\title{
Domain walls and CP violation with left right supersymmetry: implications for leptogenesis and electron EDM
}

\author{
P. Banerjee ${ }^{1}$ and U.A. Yajnik \\ Department of Physics, Indian Institute of Technology, \\ Bombay, India \\ E-mail: banerjee.piyali3@gmail.com, yajnik@iitb.ac.in
}

ABSTRACT: Low scale leptogenesis scenarios are difficult to verify due to our inability to relate the parameters involved in the early universe processes with the low energy or collider observables. Here we show that one can in principle relate the parameters giving rise to the transient $C P$ violating phase involved in leptogenesis with those that can be deduced from the observation of electric dipole moment (EDM) of the electron. We work out the details of this in the context of the left right symmetric supersymmetric model (LRSUSY) which provides a strong connection between such parameters. In particular, we show that baryon asymmetry requirements imply the scale $M_{B-L}$ of $\mathrm{U}(1)_{B-L}$ symmetry breaking to be larger than $10^{4.5} \mathrm{GeV}$. Moreover the scale $M_{R}$ of $\mathrm{SU}(2)_{R}$ symmetry breaking is tightly constrained to lie in a narrow band significantly below $M_{B-L}^{2} / M_{E W}$. These are the most stringent constraints on the parameter space of LRSUSY model being considered.

Keywords: Cosmology of Theories beyond the SM, CP violation, Supersymmetric Standard Model, Discrete Symmetries

ARXIV EPRINT: 2012.05872

\footnotetext{
${ }^{1}$ Corresponding author.
} 


\section{Contents}

1 Introduction 1

2 The supersymmetric left-right symmetric model 4

2.1 Supersymmetry preserving vacua above the electroweak scale 4

2.2 Soft terms 8

$\begin{array}{lll}2.3 & \text { The alternative vacua } & 8\end{array}$

$\begin{array}{lll}3 & \text { Vacuum structure in the early universe } & 9\end{array}$

4 Solving the Euler-Lagrange equations for Higgs vevs 12

5 Electron EDM constraints on LRSUSY 13

$6 \quad$ Leptogenesis constraints on LRSUSY 17

$\begin{array}{lll}7 & \text { Primordial gravitational wave signature } & 21\end{array}$

8 Discussion and conclusion $\quad 22$

$\begin{array}{ll}\text { A Euler-Lagrange equations for spatially varying Higgs vevs } & 24\end{array}$

B Temperature dependent masses of bidoublet Higgs scalars 25

\section{Introduction}

One of the three necessary Sakharov conditions for dynamical generation of baryon asymmetry of the universe is CP violation [1]. The Standard Model (SM) of particle physics has the ingredients to satisfy all the three Sakharov conditions and could, in principle, generate some baryon asymmetry [2]. However the requirement of a strong first order Electroweak Phase Transition (EWPT) requires the mass of the Higgs to remain less than about $80 \mathrm{GeV}[3-5]$ which is far below the mass of the recently discovered SM Higgs boson $[6,7]$. Furthermore the CP violation in the CKM matrix of SM is too small to produce a reasonably large baryon asymmetry [8].

The left right symmetric model of Mohapatra and Senjanović [9] (LRSM) is a minimal extension of the Standard Model based on the gauge group $\mathrm{SU}(3)_{c} \times \mathrm{SU}(2)_{L} \times \mathrm{SU}(2)_{R} \times$ $\mathrm{U}(1)_{B-L}$ augmented with the discrete $Z_{2}$ left-right symmetry. The usual $\mathrm{SU}(2)_{L}$ Higgs doublet of $\mathrm{SM}$ is extended to a $\mathrm{SU}(2)_{L} \times \mathrm{SU}(2)_{R}$ bidoublet. The model naturally accommodates the parity violation of SM as a result of spontaneous symmetry breaking of $\mathrm{SU}(2)_{R} \times \mathrm{U}(1)_{B-L}$ and also elegantly explains small neutrino masses via the seesaw mechanism. Both goals are achieved by adding new heavy Higgs $\mathrm{SU}(2)$ triplets in the theory. 
The supersymmetric extension of LRSM, which we call the Simplified Left Right Symmetric Supersymmetric Standard Model (SLRSUSY), marries the desirable features of both LRSM and supersymmetry. However it suffers from the drawback that spontaneous parity violation cannot take place without violating R-parity also [10]. To remedy this, one can take the supersymmetry breaking scale $M_{S}$ to be larger than the $\mathrm{SU}(2)_{R} \times \mathrm{U}(1)_{B-L}$ breaking scale $M_{B-L}$ but that would lose out on several desirable features of supersymmetry. Alternatively, one can introduce additional heavy Higgs $\mathrm{SU}(2)$ triplets with appropriate gauge group charges. This was done in the two papers [11, 12], which we shall call the Left Right Symmetric Supersymmetric Standard Model (LRSUSY). The LRSUSY model contains two distinct high mass scales, $M_{R}$ the scale of $\mathrm{SU}(2)_{R}$ symmetry breaking, and $M_{B-L}$ the scale of $\mathrm{U}(1)_{B-L}$ symmetry breaking satisfying $M_{R} \gg M_{B-L}$, an intermediate SUSY breaking scale $M_{S}$ and a low mass scale $M_{H}$ where the bidoublet Higgs get vevs. Thus, the LRSUSY model has several desirable features not possessed by SLRSUSY like spontaneous parity violation without R-parity violation, low scale supersymmetry with exact R-parity and existence of a stable lightest supersymmetric particle.

Breaking of the $Z_{2}$ discrete symmetry of left-right symmetric models in the early universe ensures the occurrence of domain walls [13, 14], but also begs for the $Z_{2}$ symmetry to be not exact to avoid conflict with the observed homogeneous universe. However an approximate $Z_{2}$ is adequate to generate the domain walls which are a robust topological prediction independent of details of parameters, and can play the same role as the phase transition bubble walls for the purpose of leptogenesis.

In the context of non-supersymmetric LRSM, the fate of the $C P$ violating phase was studied in [15] for the purpose of explaining leptogenesis. It was shown that a spatially varying $\mathrm{CP}$ violating phase occurs inside the domain walls separating the left handed and right handed domains. The observed baryon asymmetry of the universe then requires the Yukawa coupling of the right handed neutrino to the SU(2)-triplet Higgs to be larger than $10^{-2}$. The scenario also obtains heuristic constraints on the mass of the left handed neutrinos or alternatively, the temperature scale of LR symmetry breaking. Since the LRSUSY model has additional Higgs bosons as well as additional CP violating phases, it may be able to generate the necessary conditions for successful baryogenesis in the early universe. The first step in this direction was taken in [16] by showing the possibility of having a spatially varying CP violating phase in the domain walls in the context of LRSUSY. However they did not provide any quantitative estimates and left open the question of whether LRSUSY is actually capable of generating the baryon asymmetry of the universe.

The core idea of such proposals is that the spatially varying phase implies a spatially varying complex mass for left handed neutrinos inside the wall. Studying the diffusion equation for lepton number density with spatially varying complex mass results in the preferential transmission of left handed neutrinos across a slowly moving thick domain wall. The moving wall encroaches upon the energetically disfavoured right handed domain. We solve the diffusion equation numerically for various wall speeds and thicknesses, obtaining excesses of almost massless left handed neutrinos inside the left handed domain. After the 
wall disappears, electroweak sphalerons convert a part of the neutrino excess to baryon excess. We calculate the amount of baryon excess that survives the washout processes. Requiring the surviving baryon excess to be not much above the experimental limit of around $6 \times 10^{-10}$ for the baryon asymmetry to entropy ratio [17] allows us to constrain the $\left(M_{R}, M_{B-L}\right)$ parameter space of LRSUSY.

A smoking gun signature of $\mathrm{CP}$ violation in a theory is the presence of a non-zero EDM of the electron and neutron. The Standard Model predicts a non-zero electron EDM at the

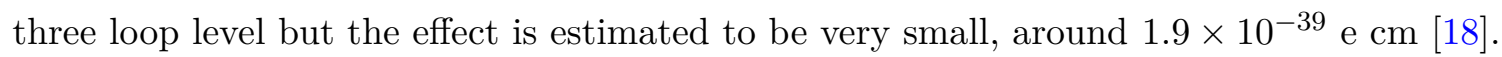
This is way below the current experimental upper bound of $1.1 \times 10^{-29} \mathrm{e} \mathrm{cm}$ obtained by the ACME II experiment [19]. Left right symmetric theories contain many additional sources of CP violation as compared to the Standard Model, and so predict larger electron and neutron EDMs. Thus the experimental bound on EDMs serve to constrain the parameters of these theories. Electron and neutron EDMs in the non-supersymmetric LRSM have been studied in several earlier works e.g. [20, 21], obtaining lower bounds on the scale $M_{B-L}$ of $\mathrm{SU}(2)_{R} \times \mathrm{U}(1)_{B-L}$ symmetry breaking and on the mass $M_{H^{\prime}}$ of the heavier scalar Higgs in the two Higgs doublets arising from breaking of left right symmetry in the bidoublet Higgs of LRSM. Electron and neutron EDMs have been studied in the SLRSUSY model by [22, 23], obtaining bounds on the masses of certain superpartners and some other parameters of SLRSUSY.

The LRSUSY model [12] contains essentially only one major unknown, the $M_{R}$ scale and a trilinear Higgs coupling parameter $\alpha$ that is used to ensure that only two out of four SM type Higgs doublets arising after $\mathrm{SU}(2)_{R} \times \mathrm{U}(1)_{B-L}$ symmetry breaking remain lighter, with a relative phase between their vacuum expectation values. In this paper we study the CP violating phase of the bidoublet fields in LRSUSY by setting up the domain wall solutions. The parameters relevant for the domain wall solutions are understood to involve the high temperature corrections needed in the early Universe, at the temperature $T_{B-L} \sim$ $M_{B-L}$. We separately investigate the contribution of the lighter two mass eigenstates of the bidoublets to the electron EDM at zero temperature, which differ only by the $T_{B-L}$ corrections. We compute the contribution of this low energy phase to the EDM at one loop and two loop levels as a function of $\alpha$. The two loop computation follows along the lines of the seminal work of Barr and Zee [24] on the electron EDM in multi Higgs doublet models. A similar calculation of electron EDM arising as a residual effect of domain wall collapse in two Higgs doublet models was done in [25]. It turns out that successful leptogenesis in LRSUSY requires $\alpha \gtrsim 0.1$. Combining this with the requirement that the EDM obtained be less than the experimental limit of $1.1 \times 10^{-29} \mathrm{e} \mathrm{cm}$, we get an allowed region in the $\left(M_{B-L}, M_{R}\right)$-parameter space of LRSUSY.

It turns out that the limit on the parameter space arising from baryon asymmetry is more stringent than the limit from electron EDM. Further requiring that the observed baryon asymmetry be explained to within an order of magnitude by LRSUSY puts stringent constraints on the $\left(M_{B-L}, M_{R}\right)$-parameter space of LRSUSY. In particular $M_{B-L}<10^{4.5} \mathrm{GeV}$ is ruled out. These are the most stringent constraints on the parameter space of LRSUSY by far. 


\section{The supersymmetric left-right symmetric model}

The gauge group of LRSUSY is the left-right symmetric group $\mathrm{SU}(3)_{c} \times \mathrm{SU}(2)_{L} \times \mathrm{SU}(2)_{R} \times$ $\mathrm{U}(1)_{B-L}$. The electric charge is then given by $Q=T_{3 L}+T_{3 R}+\frac{B-L}{2}$. The nonsupersymmetric model [9] which also elegantly permits see-saw mechanism for neutrino masses contained two triplet scalar fields of the type $\Delta$ below and one bidoublet $\Phi$ to break the electroweak symmetry. It was realised in [10] that a supersymmetric extension of this requires adding two more $\Delta$ triplets and one more $\Phi$ to cancel the anomalies, but that this leads to vacua that necessarily break electromagnetism while breaking parity. This can be repaired by adding new triplets $\Omega$ which help to break the $\mathrm{SU}(2)_{R}$ but are neutral under $B-L$ charge and save the electromagnetic charge from being broken in the vacuum [11]. Accordingly the Higgs sector consists of three left handed Higgs triplets $\Delta$, $\bar{\Delta}$ and $\Omega$, and three right handed Higgs triplets $\Delta_{c}, \bar{\Delta}_{c}$ and $\Omega_{c}$ and two Higgs bidoublets $\Phi_{1}$ and $\Phi_{2}$. They are assigned gauge group charges

$$
\begin{aligned}
\Phi_{1} & =\left(1,2,2^{*}, 0\right), & \Phi_{2} & =\left(1,2,2^{*}, 0\right), & & \\
\Omega & =(1,3,1,0), & \Delta & =(1,3,1,2), & \bar{\Delta} & =(1,3,1,-2), \\
\Omega_{c} & =\left(1,1,3^{*}, 0\right), & \Delta_{c} & =\left(1,1,3^{*},-2\right), & \bar{\Delta}_{c} & =\left(1,1,3^{*}, 2\right) .
\end{aligned}
$$

The group action is given by

$$
\begin{aligned}
\left\{\Phi_{1}, \Phi_{2}\right\} & \rightarrow U_{L}\left\{\Phi_{1}, \Phi_{2}\right\} U_{R}^{\dagger}, \\
\{\Delta, \bar{\Delta}, \Omega\} & \rightarrow U_{L}\{\Delta, \bar{\Delta}, \Omega\} U_{L}^{\dagger}, \\
\left\{\Delta_{c}, \bar{\Delta}_{c}, \Omega_{c}\right\} & \rightarrow U_{R}^{*}\left\{\Delta_{c}, \bar{\Delta}_{c}, \Omega_{c}\right\} U_{R}^{T} .
\end{aligned}
$$

It should be noted that this LRSUSY embeds naturally into a partially unified SUSY model like Pati-Salam with gauge group $\mathrm{SU}(4)_{c} \times \mathrm{SU}(2)_{L} \times \mathrm{SU}(2)_{R}$ [26]. The LH Higgs triplets $\Delta, \bar{\Delta}$ with opposite $\mathrm{U}(1)_{B-L}$ charges descend from the $(15,3,1)$ representation, whereas the RH Higgs triplets $\Delta_{c}, \bar{\Delta}_{c}$ descend from the $(15,1,3)$ representation of the Pati-Salam gauge group. The bidoublets remain as they are in Pati-Salam.

The above partial unification into Pati-Salam can be further UV completed to GUT unification into SO(10) SUSY [26]. The $(15,3,1)$ and $(15,1,3)$ representations of PatiSalam embed into a single 210 representation of $\mathrm{SO}(10)$. The bidoublet of Pati-Salam embeds into the 10 representation of $\mathrm{SO}(10)$. The embeddings described above also allow a direct UV completion of LRSUSY into SO(10) SUSY. Most routes of breakdown of the unified models to electroweak scale necessarily pass through the simpler left-right symmetric case discussed here, with exact symmetry derived from the grand unified theory. Cosmological signature such as matter-anti-matter asymmetry will be dominated by the last major events in the thermal bath and the laboratory signatures like the electron EDM will likewise be dominated by the lowest scale version of the model and that is what we have undertaken to study.

\subsection{Supersymmetry preserving vacua above the electroweak scale}

The vacuum structure of the model needed to arrive at the MSSM was analysed in Aulakh et al. [12]. We shall mostly be working at a cosmological epoch above the electroweak scale, as 
such that formalism is directly applicable. The Higgs part of the superpotential is given by

$$
\begin{aligned}
W= & m_{\Delta}\left(\operatorname{Tr} \Delta \bar{\Delta}+\Delta_{c} \bar{\Delta}_{c}\right)+\frac{m_{\Omega}}{2}\left(\operatorname{Tr} \Omega^{2}+\operatorname{Tr} \Omega_{c}^{2}\right)+\mu_{i j} \operatorname{Tr} \tau_{2} \Phi_{i}^{T} \tau_{2} \Phi_{j} \\
& +a\left(\operatorname{Tr} \Delta \Omega \bar{\Delta}+\operatorname{Tr} \Delta_{c} \Omega_{c} \bar{\Delta}_{c}\right)+\alpha_{i j}\left(\operatorname{Tr} \Omega \Phi_{i} \tau_{2} \Phi_{j}^{T} \tau_{2}+\operatorname{Tr} \Omega_{c} \Phi_{i}^{T} \tau_{2} \Phi_{j} \tau_{2}\right)
\end{aligned}
$$

where $\alpha_{i j}, \mu_{i j}$ are complex numbers satisfying $\mu_{12}=\mu_{21}, \alpha_{i j}=-\alpha_{j i}$. Define $\alpha=\alpha_{12}$. Then $\alpha_{21}=-\alpha$ and $\alpha_{11}=\alpha_{22}=0$.

The vev's of the Higgs fields permitted by the condition of preservation of electromagnetic charge are as below and will be shown to be consistently obtainable from the superpotential.

$$
\begin{aligned}
& \left\langle\Phi_{1}\right\rangle=\left(\begin{array}{cc}
k_{1} & 0 \\
0 & k_{1}^{\prime}
\end{array}\right), \quad\left\langle\Phi_{2}\right\rangle=\left(\begin{array}{cc}
k_{2} & 0 \\
0 & k_{2}^{\prime}
\end{array}\right), \\
& \langle\Omega\rangle=\left(\begin{array}{cc}
\omega & 0 \\
0 & -\omega
\end{array}\right), \quad\langle\Delta\rangle=\left(\begin{array}{cc}
0 & 0 \\
d & 0
\end{array}\right), \quad\langle\bar{\Delta}\rangle=\left(\begin{array}{cc}
0 & \bar{d} \\
0 & 0
\end{array}\right), \\
& \left\langle\Omega_{c}\right\rangle=\left(\begin{array}{cc}
\omega_{c} & 0 \\
0 & -\omega_{c}
\end{array}\right), \quad\left\langle\Delta_{c}\right\rangle=\left(\begin{array}{cc}
0 & 0 \\
d_{c} & 0
\end{array}\right), \quad\left\langle\bar{\Delta}_{c}\right\rangle=\left(\begin{array}{cc}
0 & \bar{d}_{c} \\
0 & 0
\end{array}\right),
\end{aligned}
$$

where the quantities above are in general complex numbers. We can use the $B-L$ gauge invariance to ensure that $d, \bar{d}$ have the same phase. Subsequently using $\mathrm{SU}(2)_{L}$ invariance, we can ensure that $d=\bar{d}$ and are real positive.

Next we need the $D$ terms and the $F$ terms to obtain the supersymmetry preserving vacua. The $D$-terms are given by (where $m=1,2,3$ refer to the three generators of $\mathrm{SU}(2))[10]$

$$
\begin{aligned}
D_{L, m} & =\operatorname{Tr}\left(2 \Omega^{\dagger} \tau_{m} \Omega+2 \Delta^{\dagger} \tau_{m} \Delta+2 \bar{\Delta}^{\dagger} \tau_{m} \bar{\Delta}+\Phi_{1}^{\dagger} \tau_{m} \Phi_{1}+\Phi_{2}^{\dagger} \tau_{m} \Phi_{2}\right) \\
D_{R, m} & =\operatorname{Tr}\left(2 \Omega_{c}^{\dagger} \tau_{m} \Omega_{c}+2 \Delta_{c}^{\dagger} \tau_{m} \Delta_{c}+2 \bar{\Delta}_{c}^{\dagger} \tau_{m} \bar{\Delta}_{c}+\Phi_{1} \tau_{m}^{T} \Phi_{1}^{\dagger}+\Phi_{2} \tau_{m}^{T} \Phi_{2}^{\dagger}\right) \\
D_{B-L} & =\operatorname{Tr}\left(2 \Delta^{\dagger} \Delta-2 \bar{\Delta}^{\dagger} \bar{\Delta}-2 \Delta_{c}^{\dagger} \Delta_{c}+2 \bar{\Delta}_{c}^{\dagger} \bar{\Delta}_{c}\right) .
\end{aligned}
$$

After substituting the vevs into the $D$-term expressions we get

$$
\begin{aligned}
\left\langle D_{B-L}\right\rangle & =2\left(|d|^{2}-|\bar{d}|^{2}-\left|d_{c}\right|^{2}+\left|\bar{d}_{c}\right|^{2}\right), \\
\left\langle D_{L, 1}\right\rangle & =\left\langle D_{L, 2}\right\rangle=\left\langle D_{R, 1}\right\rangle=\left\langle D_{R, 2}\right\rangle=0, \\
\left\langle D_{L, 3}\right\rangle & =2\left(-|d|^{2}+|\bar{d}|^{2}\right)+\left|k_{1}\right|^{2}-\left|k_{1}^{\prime}\right|^{2}+\left|k_{2}\right|^{2}-\left|k_{2}^{\prime}\right|^{2}, \\
\left\langle D_{R, 3}\right\rangle & =2\left(-\left|d_{c}\right|^{2}+\left|\bar{d}_{c}\right|^{2}\right)+\left|k_{1}\right|^{2}-\left|k_{1}^{\prime}\right|^{2}+\left|k_{2}\right|^{2}-\left|k_{2}^{\prime}\right|^{2} .
\end{aligned}
$$

We see that in the absence of soft terms, the energy scales of the left and the right sectors and in turn of the electroweak sector can remain independent of each other provided we solve the $D_{B-L}=0$ condition by choosing $|d|=|\bar{d}|$ and $\left|d_{c}\right|=\left|\bar{d}_{c}\right|$. Then the vanishing of $D_{L, 3}$ and $D_{R, 3}$ requires $\left|k_{1}\right|^{2}+\left|k_{2}\right|^{2}=\left|k_{1}^{\prime}\right|^{2}+\left|k_{2}^{\prime}\right|^{2}$. We shall see below a stronger condition from the vanishing of $F$ terms. 
As for the $F$ terms, to the terms already given in [12] we add the bidoublet $F$ terms:

$$
\begin{aligned}
& F_{\bar{\Delta}}=m_{\Delta} \Delta+a\left(\Delta \Omega-\frac{\operatorname{Tr} \Delta \Omega}{2}\right), \\
& F_{\Delta}=m_{\Delta} \bar{\Delta}+a\left(\Omega \bar{\Delta}-\frac{\operatorname{Tr} \Omega \bar{\Delta}}{2}\right), \\
& F_{\Omega}=m_{\Omega} \Omega+a\left(\bar{\Delta} \Delta-\frac{\operatorname{Tr} \bar{\Delta}}{2}\right)+\alpha\left(\Phi_{1} \tau_{2} \Phi_{2}^{T} \tau_{2}-\Phi_{2} \tau_{2} \Phi_{1}^{T} \tau_{2}\right), \\
& F_{\overline{\Delta_{c}}}=m_{\Delta} \Delta_{c}+a\left(\Delta_{c} \Omega_{c}-\frac{\operatorname{Tr} \Delta_{c} \Omega_{c}}{2}\right), \\
& F_{\Delta_{c}}=m_{\Delta} \bar{\Delta}_{c}+a\left(\Omega_{c} \bar{\Delta}_{c}-\frac{\operatorname{Tr} \Omega_{c} \overline{\Delta_{c}}}{2}\right), \\
& F_{\Omega_{c}}=m_{\Omega} \Omega_{c}+a\left(\bar{\Delta}_{c} \Delta_{c}-\frac{\operatorname{Tr} \bar{\Delta}_{c} \Delta_{c}}{2}\right)+\alpha\left(\Phi_{1}^{T} \tau_{2} \Phi_{2} \tau_{2}-\Phi_{2}^{T} \tau_{2} \Phi_{1} \tau_{2}\right), \\
& F_{\Phi_{1}}=2 \mu_{11} \tau_{2} \Phi_{1}^{T} \tau_{2}+2 \mu_{12} \tau_{2} \Phi_{2}^{T} \tau_{2}+\alpha\left(\tau_{2} \Phi_{2}^{T} \tau_{2} \Omega-\tau_{2} \Phi_{2}^{T} \Omega^{T} \tau_{2}+\tau_{2} \Omega_{c} \Phi_{2}^{T} \tau_{2}-\Omega_{c}^{T} \tau_{2} \Phi_{2}^{T} \tau_{2}\right), \\
& F_{\Phi_{2}}=2 \mu_{12} \tau_{2} \Phi_{1}^{T} \tau_{2}+2 \mu_{22} \tau_{2} \Phi_{2}^{T} \tau_{2}-\alpha\left(\tau_{2} \Phi_{1}^{T} \tau_{2} \Omega-\tau_{2} \Phi_{1}^{T} \Omega^{T} \tau_{2}+\tau_{2} \Omega_{c} \Phi_{1}^{T} \tau_{2}-\Omega_{c}^{T} \tau_{2} \Phi_{1}^{T} \tau_{2}\right) .
\end{aligned}
$$

After substituting the vevs, the expressions for the F-terms become

$$
\begin{aligned}
& \left\langle F_{\bar{\Delta}}\right\rangle=\left(\begin{array}{cc}
0 & 0 \\
d\left(m_{\Delta}+a \omega\right) & 0
\end{array}\right), \\
& \left\langle F_{\Delta}\right\rangle=\left(\begin{array}{cc}
0 & \bar{d}\left(m_{\Delta}+a \omega\right) \\
0 & 0
\end{array}\right), \\
& \left\langle F_{\Omega}\right\rangle=\left(\begin{array}{cc}
m_{\Omega} \omega+\frac{a d \bar{d}}{2}+\alpha\left(k_{1} k_{2}^{\prime}-k_{1}^{\prime} k_{2}\right) & 0 \\
0 & -\left(m_{\Omega} \omega+\frac{a d \bar{d}}{2}+\alpha\left(k_{1} k_{2}^{\prime}-k_{1}^{\prime} k_{2}\right)\right)
\end{array}\right), \\
& \left\langle F_{\bar{\Delta}_{c}}\right\rangle=\left(\begin{array}{cc}
0 & d_{c}\left(m_{\Delta}+a \omega_{c}\right) \\
0 & 0
\end{array}\right), \\
& \left\langle F_{\Delta_{c}}\right\rangle=\left(\begin{array}{cc}
0 & 0 \\
\bar{d}_{c}\left(m_{\Delta}+a \omega_{c}\right) & 0
\end{array}\right) \text {, } \\
& \left\langle F_{\Omega_{c}}\right\rangle=\left(\begin{array}{cc}
m_{\Omega} \omega_{c}+\frac{a d_{c} \bar{d}_{c}}{2}+\alpha\left(k_{1} k_{2}^{\prime}-k_{1}^{\prime} k_{2}\right) & 0 \\
0 & -\left(m_{\Omega} \omega_{c}+\frac{a d_{c} \bar{d}_{c}}{2}+\alpha\left(k_{1} k_{2}^{\prime}-k_{1}^{\prime} k_{2}\right)\right)
\end{array}\right), \\
& \left\langle F_{\Phi_{1}}\right\rangle=\left(\begin{array}{cc}
2 \mu_{11} k_{1}^{\prime}+2 \mu_{12} k_{2}^{\prime}+2 \alpha k_{2}^{\prime}\left(\omega-\omega_{c}\right) & 0 \\
0 & 2 \mu_{11} k_{1}+2 \mu_{12} k_{2}-2 \alpha k_{2}\left(\omega-\omega_{c}\right)
\end{array}\right) \text {, } \\
& \left\langle F_{\Phi_{2}}\right\rangle=\left(\begin{array}{cc}
2 \mu_{12} k_{1}^{\prime}+2 \mu_{22} k_{2}^{\prime}-2 \alpha k_{1}^{\prime}\left(\omega-\omega_{c}\right) & 0 \\
0 & 2 \mu_{12} k_{1}+2 \mu_{22} k_{2}+2 \alpha k_{1}\left(\omega-\omega_{c}\right)
\end{array}\right) .
\end{aligned}
$$

Since the primary goal of previous analyses was to ensure supersymmetry preserving vacuum above the electroweak scale, the bidoublet VEVs were not relevant. However we shall be interested in domain walls for the purpose of leptogenesis. Here the bidoublet condensates appear transitorily in the interiors of the domain walls and so we set up the energetics for their vevs. But away from the walls we wish to recover the electroweak 
symmetry preserving vacuum. Hence we investigate all the fields for their permitted vevs that ensure $D$ and $F$-flatness. For generic values of $\mu_{11}, \mu_{12}$ and $\mu_{22}$, the entries of $F_{\Phi_{1}}$ and $F_{\Phi_{2}}$ are linearly independent. This is also true for the approximate fine tuning of $\mu_{12}$ that is discussed in more detail later on. Hence flatness of $F_{\Phi_{1}}$ and $F_{\Phi_{2}}$ implies that the vevs of $k_{1}, k_{1}^{\prime}, k_{2}, k_{2}^{\prime}$ are all zero consistent with unbroken electroweak symmetry. This also automatically ensures the vanishing of $D_{L, 3}$ and $D_{R, 3}$ in eqs. (2.6). We can now invoke the detailed analysis of Aulakh et al. [12] in order to obtain the complete solution set of supersymmetry satisfying vacua and justify their stability against radiative corrections.

The model extended with the inclusion of the $\Omega$ triplets gives rise to two independent physical scales, $M_{R}$ at which $\mathrm{SU}(2)_{R} \rightarrow \mathrm{U}(1)_{R}$ and separately $M_{B-L}$ of $\mathrm{U}(1)_{B-L}$ breaking. The two scales are determined in terms of the vevs by

$$
\begin{aligned}
M_{R} & \cong \frac{m_{\Delta}}{-a} \\
M_{B-L} & \cong \frac{\sqrt{2 m_{\Omega} m_{\Delta}}}{-a}
\end{aligned}
$$

(note $a<0$ ). At this point, anticipating our later discussion on alternative vacua, we note that one can also have $\mathrm{SU}(2)_{R}$ preserving and $\mathrm{SU}(2)_{L} \rightarrow \mathrm{U}(1)_{L}$ vacuum with the corresponding alternative vevs described later. Returning to the above relations, we are faced with the scale of $\mathrm{U}(1)_{B-L}$ breaking unrelated to any other scale, which can be traced to the new mass parameter $M_{\Omega}$ in the superportential eq. (2.3). A solution to this proliferation of mass scales was sought in [12] by invoking an $R$ symmetry of the superpotential which forbids the terms $\Omega^{2}$ and $\Omega_{c}^{2}$. The $R$ charge values can be set to

$$
\begin{aligned}
\Delta, \bar{\Delta}, \Delta_{c}, \bar{\Delta}_{c}, \Phi_{i} & \rightarrow 1 \\
\Omega & \rightarrow 0 \\
L, L_{c}, Q, Q_{c} & \rightarrow \frac{1}{2}
\end{aligned}
$$

where the $L, Q$ etc are matter superfields which are not relevant to this paper. The terms $\Omega^{2}$ and $\Omega_{c}^{2}$ can then be introduced only as soft terms, with the coefficients $m_{\Omega}=m_{\Omega_{c}}$ determined by SUSY breaking scale $\cong M_{E W}$. This leads to an elegant simplification giving rise to the see-saw relation

$$
M_{B-L}^{2} \cong M_{R} M_{E W}
$$

To summarise, we see that there are two possible degenerate vacua LH type and RH type described explicitly later on. All the neutral and charged Higgs supermultiplets arising from $\Delta, \Delta_{c}, \bar{\Delta}, \bar{\Delta}_{c}$ have masses around $M_{R}$ in both types of domains. In both domains half of the charged and neutral Higgs supermultiplets arising from the bidoublets $\Phi_{1}, \Phi_{2}$ have small masses near electroweak scale due to the fine tuning of $\mu_{12}$ described in more detail below; the other half have masses near $M_{R}$. In the left domain, the charged Higgs supermultiplets arising from $\Omega_{c}$ have masses around $M_{R}$ while the neutral Higgs supermultiplets have masses around $M_{B-L}$. The Higgs supermultiplets arising from $\Omega$ have masses around $M_{B-L}^{2} / M_{R}$. The L-R flipped fields get vev's in the right domain at numerically the same mass scales. Detailed mass formulas can be found in [12]. 


\subsection{Soft terms}

To the SUSY scalar potential

$$
\begin{aligned}
V_{\mathrm{SUSY}}= & \left|F_{\bar{\Delta}}\right|^{2}+\left|F_{\Delta}\right|^{2}+\left|F_{\bar{\Delta}_{c}}\right|^{2}+\left|F_{\Delta_{c}}\right|^{2}+\left|F_{\Omega}\right|^{2}+\left|F_{\Omega_{c}}\right|^{2}+\left|F_{\Phi_{1}}\right|^{2}+\left|F_{\Phi_{2}}\right|^{2} \\
& +\sum_{m=1}^{3}\left(\left|D_{L, m}\right|^{2}+\left|D_{R, m}\right|^{2}\right)+\left|D_{B-L}\right|^{2},
\end{aligned}
$$

we add the following soft mass terms for the bidoublets

$$
\begin{aligned}
V_{\text {soft }}= & -\mu_{1}^{2} \operatorname{Tr}\left(\Phi_{1}^{\dagger} \Phi_{1}\right)-\mu_{2}^{2} \operatorname{Tr}\left(\Phi_{2}^{\dagger} \Phi_{2}\right) \\
& -e^{i \beta_{3}} \mu_{3}^{2} \operatorname{Tr}\left(\Phi_{1}^{\dagger} \tau_{2} \Phi_{1}^{*} \tau_{2}\right)-e^{i \beta_{4}} \mu_{4}^{2} \operatorname{Tr}\left(\Phi_{2}^{\dagger} \tau_{2} \Phi_{2}^{*} \tau_{2}\right)-e^{i \beta_{5}} \mu_{5}^{2} \operatorname{Tr}\left(\Phi_{1}^{\dagger} \tau_{2} \Phi_{2}^{*} \tau_{2}\right)+\text { h.c. }
\end{aligned}
$$

where $\mu_{i}^{2}>0$ and $\beta_{3}, \beta_{4}, \beta_{5}$ are explicit CP phases. Substituting the vevs we get $\langle V\rangle=$ $\left\langle V_{\mathrm{SUSY}}\right\rangle+\left\langle V_{\text {soft }}\right\rangle$, where

$$
\begin{aligned}
\left\langle V_{\mathrm{SUSY}}\right\rangle= & \left|\left\langle F_{\bar{\Delta}}\right\rangle\right|^{2}+\left|\left\langle F_{\Delta}\right\rangle\right|^{2}+\left|\left\langle F_{\bar{\Delta}_{c}}\right\rangle\right|^{2}+\left|\left\langle F_{\Delta_{c}}\right\rangle\right|^{2}+\left|\left\langle F_{\Omega}\right\rangle\right|^{2}+\left|\left\langle F_{\Omega_{c}}\right\rangle\right|^{2}+\left|\left\langle F_{\Phi_{1}}\right\rangle\right|^{2}+\left|\left\langle F_{\Phi_{2}}\right\rangle\right|^{2} \\
& +\left|\left\langle D_{L, 3}\right\rangle\right|^{2}+\left|\left\langle D_{R, 3}\right\rangle\right|^{2}+\left|\left\langle D_{B-L}\right\rangle\right|^{2},
\end{aligned}
$$

and

$$
\begin{aligned}
\left\langle V_{\text {soft }}\right\rangle= & -\mu_{1}^{2}\left(\left|k_{1}\right|^{2}+\left|k_{1}^{\prime}\right|^{2}\right)-\mu_{2}^{2}\left(\left|k_{2}\right|^{2}+\left|k_{2}^{\prime}\right|^{2}\right) \\
& -4 \mu_{3}^{2} \operatorname{Re}\left(e^{i \beta_{3}} k_{1}^{*}\left(k_{1}^{\prime}\right)^{*}\right)-4 \mu_{4}^{2} \operatorname{Re}\left(e^{i \beta_{4}} k_{2}^{*}\left(k_{2}^{\prime}\right)^{*}\right)-2 \mu_{5}^{2} \operatorname{Re}\left(e^{i \beta_{5}}\left(k_{1}^{*}\left(k_{2}^{\prime}\right)^{*}+\left(k_{1}^{\prime}\right)^{*} k_{2}^{*}\right)\right) .
\end{aligned}
$$

We shall take the fine tuning condition $\mu_{12}^{2} \approx \mu_{11} \mu_{22}+\alpha^{2} M_{R}^{2}$ of Aulakh et el. [12] which ensures that half of the neutral and charged bidoublet Higgs scalars have masses near zero and the other half have masses near $M_{R}$. Our soft masses $\mu_{1}, \ldots, \mu_{5}$ are chosen to be around $\alpha^{2} M_{R}$, so that at temperatures below the SUSY breaking scale, the four light Higgs scalars eventually become the Higgs bosons of a two Higgs doublet model (2HDM) satisfying $\mathrm{SU}(3)_{c} \times \mathrm{SU}(2)_{L} \times \mathrm{U}(1)_{Y}$ gauge symmetry. At even lower temperatures, the $\mathrm{SM}$ Higgs boson arises from the $2 \mathrm{HDM}$.

\subsection{The alternative vacua}

Invoking the analysis of Aulakh et al. [12] and exploiting the left-right symmetry of the theory, the complete SUSY preserving solution set has four alternative choices for the vacuum: $\left(\omega, d, \omega_{c}, d_{c}\right)=(0,0,0,0)$, or $\left(\omega, d, \omega_{c}, d_{c}\right)=\left(\frac{m_{\Delta}}{-a}, \frac{\sqrt{2 m_{\Omega} m_{\Delta}}}{-a}, 0,0\right)$, or $\left(\omega, d, \omega_{c}, d_{c}\right)=$ $\left(0,0, \frac{m_{\Delta}}{-a}, \frac{\sqrt{2 m_{\Omega} m_{\Delta}}}{-a}\right)$, or $\left(\omega, d, \omega_{c}, d_{c}\right)=\left(\frac{m_{\Delta}}{-a}, \frac{\sqrt{2 m_{\Omega} m_{\Delta}}}{-a}, \frac{m_{\Delta}}{-a}, \frac{\sqrt{2 m_{\Omega} m_{\Delta}}}{-a}\right)$. In all the four solutions, $\left(k_{1}, k_{1}^{\prime}, k_{2}, k_{2}^{\prime}\right)=(0,0,0,0)$. Of these, the solution $\left(\omega, d, \omega_{c}, d_{c}, k_{1}, k_{1}^{\prime}, k_{2}, k_{2}^{\prime}\right)=$ $\left(\frac{m_{\Delta}}{-a}, \frac{\sqrt{2 m_{\Omega} m_{\Delta}}}{-a}, \frac{m_{\Delta}}{-a}, \frac{\sqrt{2 m_{\Omega} m_{\Delta}}}{-a}, 0,0,0,0\right)$ needs to be rejected as it does not provide low energy electroweak gauge symmetry. The other two non-trivial solutions should both deserve attention since they are left-right equivalents of each other. They result in the left-right symmetric gauge group $\mathrm{SU}(3)_{c} \times \mathrm{SU}(2)_{L} \times \mathrm{SU}(2)_{R} \times \mathrm{U}(1)_{B-L}$ breaking down to either the desirable Minimal Supersymmetric Standard Model $(\mathrm{MSSM}) \mathrm{SU}(3)_{c} \times \mathrm{SU}(2)_{L} \times \mathrm{U}(1)_{Y}$, or into the right-handed equivalent of the MSSM SU(3) $)_{c} \times \mathrm{SU}(2)_{R} \times \mathrm{U}(1)_{Y}$. Thus, obtaining the physically relevant vacuum is a challenge within this model. A well recognised solution 
is the presence of a gauge singlet scalar field with its own mass scale, favouring the LH vacuum at low energies [27]. The consequences of avoiding exact left-right parity through the spontaneous breaking route are being extensively studied [28-30]. In the supersymmetric case a more appealing alternative is for its physics to be tied to supersymmetry breaking in the hidden sector [31].

From the cosmological viewpoint, the early universe enters an epoch with two types of domains. In the left handed ( $\mathrm{LH})$ domains, the right handed vevs take non-zero values and in the right handed $(\mathrm{RH})$ domains, the left handed vevs take non-zero values. The corresponding SUSY preserving vevs are

$$
\begin{aligned}
& \left(\omega, d, \omega_{c}, d_{c}, k_{1}, k_{1}^{\prime}, k_{2}, k_{2}^{\prime}\right)=\left(0,0, \frac{m_{\Delta}}{-a}, \frac{\sqrt{2 m_{\Omega} m_{\Delta}}}{-a}, 0,0,0,0\right) \quad \text { LH domain, } \\
& \left(\omega, d, \omega_{c}, d_{c}, k_{1}, k_{1}^{\prime}, k_{2}, k_{2}^{\prime}\right)=\left(\frac{m_{\Delta}}{-a}, \frac{\sqrt{2 m_{\Omega} m_{\Delta}}}{-a}, 0,0,0,0,0,0\right) \quad \text { RH domain. }
\end{aligned}
$$

The formation of the two types of domains also leads to topological domain walls separating them $[13,14,32,33]$. This can be traced to the presence of causal horizons and the irreversibility of time evolution in the context of Big Bang cosmology. The presence of these walls or energy barriers conflicts with current cosmology. Several earlier works have discussed how such walls could have disappeared fast enough so as to be consistent with present day observations [31]. The older study [16] demonstrated the existence of domain walls containing a CP violating phase in LRSUSY with implications to leptogenesis, but only as a proof-of-concept study. In this paper, we extend their ideas greatly and come up with quantitative estimates relating the parameter ranges that can give rise to the required spatially varying $\mathrm{CP}$ violating phase within the domain wall with the non-zero phase required for electron EDM in zero temperature translation invariant theory. The next section provides the details.

We end the section by noting that possible conflict of existence of domain walls with standard cosmology can be avoided by assuming that there exists small effects which violate the discrete parity or left right symmetry. Thus we are able to leverage their presence for low scale leptogenesis before they disappear. Consistency with cosmology requires that the walls disappear before the Big Bang Nucleosynthesis commences. This puts a lower bound of $T \gtrsim 10 \mathrm{MeV}$ on the temperature by which the disappearance is complete. This being a much later epoch than the one at which we study the domain walls allows for minuscule effects to achieve the required disappearance. Earlier works showed that addition of tiny discrete parity violating non-renormalisable terms [31] or soft SUSY breaking terms [16] can annihilate the domain wall early enough so as not to conflict with standard cosmology.

\section{Vacuum structure in the early universe}

In this section we study the domain wall solutions possible in this model at a temperature above the electroweak scale. We shall see that, for a certain choice of soft SUSY breaking mass terms for the bidoublets, the temperature dependent effective potential makes it energetically favourable for the bidoublet fields to take non-zero vevs within the wall while 
continuing to take (almost) zero vevs outside it. Moreover, the generic $O(1)$ phases in the soft mass terms entail a consequence that the bidoublet fields take on spatially varying $C P$ violating phases inside the wall while returning to constant non-zero phases outside the wall.

We begin by recapitulating the essential features involved in setting up the temperature dependent effective potential. The temperature dependence of the squared mass term of a Higgs scalar has been evaluated at the one loop level in earlier works [34]. Let $V_{\text {tree }}=V_{\text {SUSY }}+V_{\text {soft }}$ denote the tree level Higgs potential of the LRSUSY model at zero temperature. Let $V_{\mathrm{CW}}$ denote the Coleman-Weinberg one loop contribution to the potential at zero temperature renormalised to the energy scale $M_{B-L}$, given by [35]

$$
V_{\mathrm{CW}}=\frac{1}{64 \pi^{2}} \sum_{i}(-1)^{2 s_{i}} n_{i} m_{i}(\Phi)^{4}\left(\ln \frac{m_{i}(\Phi)^{2}}{M_{B-L}^{2}}-C_{i}\right)
$$

where the sum runs over all particles $i$ in the theory, $s_{i}, n_{i}, m_{i}(\Phi)$ are the spin, degrees of freedom and field dependent mass respectively of particle $i, C_{i}$ is a renormalisation scheme dependent constant for particle $i$ and the renormalisation scale is set to $M_{B-L}$. The degrees of freedom for Higgs scalars, $W_{L}, Z_{L}$ and top quark are 1, 6, 3 and 12 respectively. The field dependent masses are obtained from the zero temperature mass matrix defined in equation (B.1) in appendix B. By adding counter terms, we assume that the parameters appearing in the potential are one-loop corrected so that $V_{\text {tree }}$ continues to describe the one-loop corrected tree level Higgs potential at zero temperature. Let $V_{1}(T)$ denote the one loop contribution and $V_{\text {daisy }}(T)$ the daisy contribution at a finite temperature $T$. Since the temperature scale $M_{B-L}$ is much higher than the masses of the lighter half of the bidoublet Higgs, left handed gauge bosons, and the top quark, we can assume that we are in the high temperature regime with respect to the above particles and the remaining particles which are heavier than $M_{B-L}$ are decoupled from the temperature dependence of the potential. In this regime, $V_{1}(T)$ is well approximated by the field dependent expression [34-36]

$$
V_{1}(T)=\sum_{i} \frac{\left(1+(-1)^{2 s_{i}}\right) n_{i}}{2}\left(\frac{m_{i}(\Phi)^{2} T^{2}}{24}+O\left(m_{i}(\Phi)^{3} T\right)\right),
$$

where the bosonic sum runs over all the neutral Higgs scalars in the lighter half of the bidoublets as well as the left handed gauge bosons $W_{L}, Z_{L}$, and the fermionic sum can be taken to only include the top quark. In computing the field dependent masses of these particles, we need to take the mass matrix corresponding the above particles only. The daisy contribution is approximated by the field dependent expression [37, 38]

$$
V_{\text {daisy }}\left(M_{B-L}\right)=-\frac{T}{12 \pi} \sum_{B^{\prime}} n_{B^{\prime}}\left(\left(\bar{m}_{B^{\prime}}(\Phi, T)^{2}\right) 3 / 2-\left(m_{B^{\prime}}(\Phi)^{2}\right)^{3 / 2}\right),
$$

where the sum runs over the Higgs bosons and longitudinal modes of gauge bosons mentioned above and $\bar{m}_{B^{\prime}}(\Phi, T)^{2}$ is the eigenvalue of the daisy corrected field dependent mass matrix which depends quadratically on $T$. The daisy corrections modify the mass matrix by $O\left(T^{2}\right)$. For high temperatures, they give rise to a cubic temperature dependent but field independent term which can overwhelm the linear temperature dependent term in equation (3.1) at higher temperatures. However, they do not enter the temperature corrections of the masses of the particles. 
For our model, the SUSY scalar potential and the soft mass terms for the bidoublets receive temperature corrections determined by the scale $M_{B-L}$. The temperature dependence of the squared mass term of a Higgs scalar has been evaluated at the one loop level in earlier works $[34,36]$ and is discussed in more detail in appendix B. It turns out that the temperature correction to each mass matrix element is about $\left(\Delta m^{2}\right)^{T} \sim O\left(g^{2} T^{2}\right)$. Thus, going from zero temperature to temperature $M_{B-L}$ entails the raising of the mass matrix elements by $O\left(g^{2} T^{2}\right)$. For the leptogenesis calculations, we work at the high temperature $T=M_{B-L}$ with the mass matrix arising from the SUSY scalar potential and the soft masses described in detail in appendix B. These choices for the mass parameters ensure that the mass matrix of the bidoublets has a negative eigenvalue inside the wall whereas all its eigenvalues are positive outside. This in turn means that it is energetically favourable for the bidoublet fields to take non-zero vevs inside the wall while continuing to take zero vevs outside, even though the soft terms have negative squared masses. For the zero temperature electron EDM calculation that we do later, we can work in a four Higgs doublet model $[39,40]$ with temperature corrections dropped from the mass matrix of appendix B.

Let the spatial coordinate giving the distance from the wall, in units of inverse temperature $1 / T$, be denoted by $x$. The fields constituting the wall have substantial variation only in a narrow region $\Delta x \sim(L / v)$ where $v$ is a generic scalar vacuum expectation value and $L^{-1} \sim \sqrt{\lambda}$ is derived from a generic quartic coupling $\lambda$ of a renormalisable field theory. The SUSY preserving vev 8-tuples inside the two domains are given by eq. (2.18). We want a smooth variation of the vev 8-tuple as a function of $x$, going from the LH domain to the $\mathrm{RH}$ domain passing through the wall on the way. By the argument in the previous section, this necessarily entails breaking SUSY inside the wall. Thus in order to get the shapes of the $x$-dependent vevs of the Higgs fields, we need to first write down a functional for the energy per unit area of the wall and then minimise the functional via Euler-Lagrange equations.

Let $\dot{f}$ denote the derivative of vev of field $f$ with respect to $x$. Let $r_{1}, i_{1}$ be the real and imaginary parts of vev of $k_{1}, r_{2}, i_{2}, \ldots, r_{2}^{\prime}, i_{2}^{\prime}$ the real and imaginary parts of the vevs of the respective bidoublet fields. We make the simplifying assumptions that the non-bidoublet Higgs fields are real everywhere. The finite temperature energy per unit area, which is the sum of gradient energies and potential energies of all the fields, plus field dependent temperature corrections can now be taken to be,

$$
\begin{aligned}
H^{T}=\int d x( & \frac{1}{2}\left(\dot{\omega}^{2}+{\dot{\omega_{c}}}^{2}+\dot{d}^{2}+\dot{\bar{d}}^{2}+{\dot{d_{c}}}^{2}+{\dot{d_{c}}}^{2}\right. \\
& \left.+{\dot{r_{1}}}^{2}+{\dot{i_{1}}}^{2}+{\dot{r_{1}^{\prime}}}^{2}+{\dot{i_{1}^{\prime}}}^{2}+{\dot{r_{2}}}^{2}+{\dot{i_{2}}}^{2}+{\dot{r_{2}^{\prime}}}^{2}+{\dot{i_{2}^{\prime}}}^{2}\right) \\
& \left.+\left\langle V_{\mathrm{SUSY}}\right\rangle+\left\langle V_{\mathrm{soft}}^{T}\right\rangle\right) .
\end{aligned}
$$

Here a superscript $T$ on $H$ and $V_{\text {soft }}$ is a reminder of the temperature dependence. We determine the domain wall solutions such that SUSY is preserved asymptotically by the vevs upto relatively small temperature correction. It is only in the narrow region of the wall where the omega fields become small that the temperature dependent terms and soft terms become more significant. In the equations below, all the vev are meant to be temperature 
dependent, though for simplicity of notation we drop the superscript $T$.

$$
\begin{aligned}
\langle\omega(-\infty)\rangle & =\langle d(-\infty)\rangle=\langle\bar{d}(-\infty)\rangle=0, \\
\left\langle\omega_{c}(-\infty)\right\rangle & =M_{R},\left\langle d_{c}(-\infty)\right\rangle=\left\langle\bar{d}_{c}(-\infty)\right\rangle=M_{B-L}, \\
\left\langle r_{1}(-\infty)\right\rangle & =\left\langle i_{1}(-\infty)\right\rangle=\left\langle r_{1}^{\prime}(-\infty)\right\rangle=\left\langle i_{1}^{\prime}(-\infty)\right\rangle=0, \\
\left\langle r_{2}(-\infty)\right\rangle & =\left\langle i_{2}(-\infty)\right\rangle=\left\langle r_{2}^{\prime}(-\infty)\right\rangle=\left\langle i_{2}^{\prime}(-\infty)\right\rangle=0
\end{aligned}
$$

in the left domain and

$$
\begin{aligned}
\left\langle\omega_{c}(\infty)\right\rangle & =\left\langle d_{c}(\infty)\right\rangle=\left\langle\bar{d}_{c}(\infty)\right\rangle=0, \\
\langle\omega(-\infty)\rangle & =M_{R},\langle d(\infty)\rangle=\langle\bar{d}(\infty)\rangle=M_{B-L}, \\
\left\langle r_{1}(\infty)\right\rangle & =\left\langle i_{1}(\infty)\right\rangle=\left\langle r_{1}^{\prime}(\infty)\right\rangle=\left\langle i_{1}^{\prime}(\infty)\right\rangle=0, \\
\left\langle r_{2}(\infty)\right\rangle & =\left\langle i_{2}(\infty)\right\rangle=\left\langle r_{2}^{\prime}(\infty)\right\rangle=\left\langle i_{2}^{\prime}(\infty)\right\rangle=0
\end{aligned}
$$

in the right domain.

With all the parameters in place, we can now minimise the energy density per unit area by solving the Euler-Lagrange equations arising from equation (3.3). The equations are given explicitly in appendix A for completeness. In the next section we describe how to solve them numerically in order to obtain the shapes of the spatially varying vevs of the Higgs fields both within and outside the wall. The vevs take the limiting values described in equations (3.4), (3.5) outside the wall.

\section{Solving the Euler-Lagrange equations for Higgs vevs}

The Euler-Lagrange equations in appendix A form a coupled system of second order nonlinear differential equations satisfying the boundary conditions of equation (3.4) in the left domain and equation (3.5) in the right domain. Since the derivatives of the vevs are zero in both left and right domains, these equations are not well-suited for numerical solution by shooting methods. Because of the large number of non-linear equations, their numerical solution also faces difficulties under finite element or path deformation [41] methods.

Naive attempts to solve the Euler-Lagrange equations as an initial value system also run into problems. This is because if we take the initial conditions at a point in the left domain, the algorithms give us the SUSY flat left domain solution only as that solution minimises the energy density to zero. A similar statement holds if we take the initial conditions at a point in the right domain. We have to somehow model the loss of translation invariance due to the domain wall in our solution.

Since $\Omega, \Omega_{c}$ have the heaviest vevs outside the wall, we fix a natural ansatz for them that smoothly goes from the LH solution to the RH solution while passing through the wall on the way. The wall is assumed to extend from $-L$ to $L$ in units of inverse temperature. The ansatz takes the form of kink functions:

$$
\begin{aligned}
\omega_{c}(x) & =\left(1-\tanh \left(\frac{m_{\Delta}}{-2 a L} x\right)\right) \frac{m_{\Delta}}{-2 a}, \\
\omega(x) & =\left(1+\tanh \left(\frac{m_{\Delta}}{-2 a L} x\right)\right) \frac{m_{\Delta}}{-2 a} .
\end{aligned}
$$


The ansatz has the property that $\omega(x), \omega_{c}(x)$ take the correct limiting values outside the wall in both domains, but are non-zero within the wall. In the example plots later on, we shall be taking $L \sim(\sqrt{\lambda})^{-1} \sim 5$ for concreteness.

Fixing the ansatz for the vevs of $\Omega, \Omega_{c}$ models the effect of the wall and reduces the Euler-Lagrange equations to a set of 12 coupled second order differential equations for the four triplet Higgs vevs $d, \bar{d}, d_{c}, \bar{d}_{c}$ and the eight vevs corresponding to the real and imaginary parts of the bidoublet Higgs fields.

With this setting we solve the Euler-Lagrange equations as an initial value problem numerically using the GSL 2.6 library, setting the stepping function to be Runge-Kutta Dormand-Prince $(8,9)$ with step size, absolute error and relative error of $10^{-6}$. The obtained solutions for the bidoublet vevs are non-zero and spatially varying inside the wall but become zero outside. For the triplet vevs the obtained solutions are spatially varying inside the wall and approach their constant SUSY determined values outside. The vevs of $\Delta, \Delta_{c}$ are very sensitive to the values of the vevs of $\Omega, \Omega_{c}$ and quickly drop to zero towards the centre of the wall as that minimises the energy density.

Figures 1 and 2 show how the vacuum expectation values of the heavy triplet Higgs fields $\Omega, \Omega_{c}, \Delta, \Delta_{c}$ vary as a function of the distance $x$ from the wall for an ad hoc setting of parameters $a=-1.5, \alpha=0.006, \mu_{11}=0.7 M_{R}, \mu_{22}=0.7 M_{R}, \mu_{1}=0.2 \alpha^{2} M_{R}, \mu_{2}=$ $0.3 \alpha^{2} M_{R}, \mu_{3}=0.5 \alpha^{2} M_{R}, \beta_{3}=-0.5, \mu_{4}=1.5 \alpha^{2} M_{R}, \beta_{4}=1.2, \mu_{5}=0.1 \alpha^{2} M_{R}, \beta_{5}=-1.4$, $M_{R}=10^{11} \mathrm{GeV}$ and $M_{B-L}=10^{6.5} \mathrm{GeV}$. Finally the generic quartic coupling $\lambda$ which can be determined from those appearing in the potential, is taken to be $\lambda \sim L^{-2}=0.04$.

Figure 3 exhibits the spatial variation of the real and imaginary parts of the vacuum expectation values of the bidoublets. It turns out that the vevs of the fields $k_{1}$ and $k_{2}$ are real throughout while $k_{1}^{\prime}$ and $k_{2}^{\prime}$ do take spatially varying complex vevs. In figure 4 , we plot the complex phases of vevs of $k_{1}^{\prime}$ and $k_{2}^{\prime}$ as a function of $x$. Observe that the phases vary inside the wall but converge to a constant non-zero value outside.

These considerations show that a spatially varying $\mathrm{CP}$ violating phase can indeed be produced by the bidoublet Higgs vevs inside the domain wall in the early universe. This phase persists as a constant non-zero quantity outside the wall. In the next two sections, we investigate the implications of this phenomenon for the electric dipole moment of the electron and the baryon asymmetry of the universe.

\section{Electron EDM constraints on LRSUSY}

The zero temperature mass matrix of the neutral components of the bidoublet Higgs fields is given in appendix B. As there are two neutral complex components in each bidoublet, we get in total eight real neutral fields and so the mass matrix is $8 \times 8$. It turns out that the neutral mass eigenstates induce complex phases for the bidoublet Higgs fields relative to each other. This is true both within the wall as well as outside it. This feature gives rise to an electric dipole moment (EDM) for the electron at one loop and two loop levels. The maximum effect on the electron EDM is exerted by the lightest mass eigenstate. 

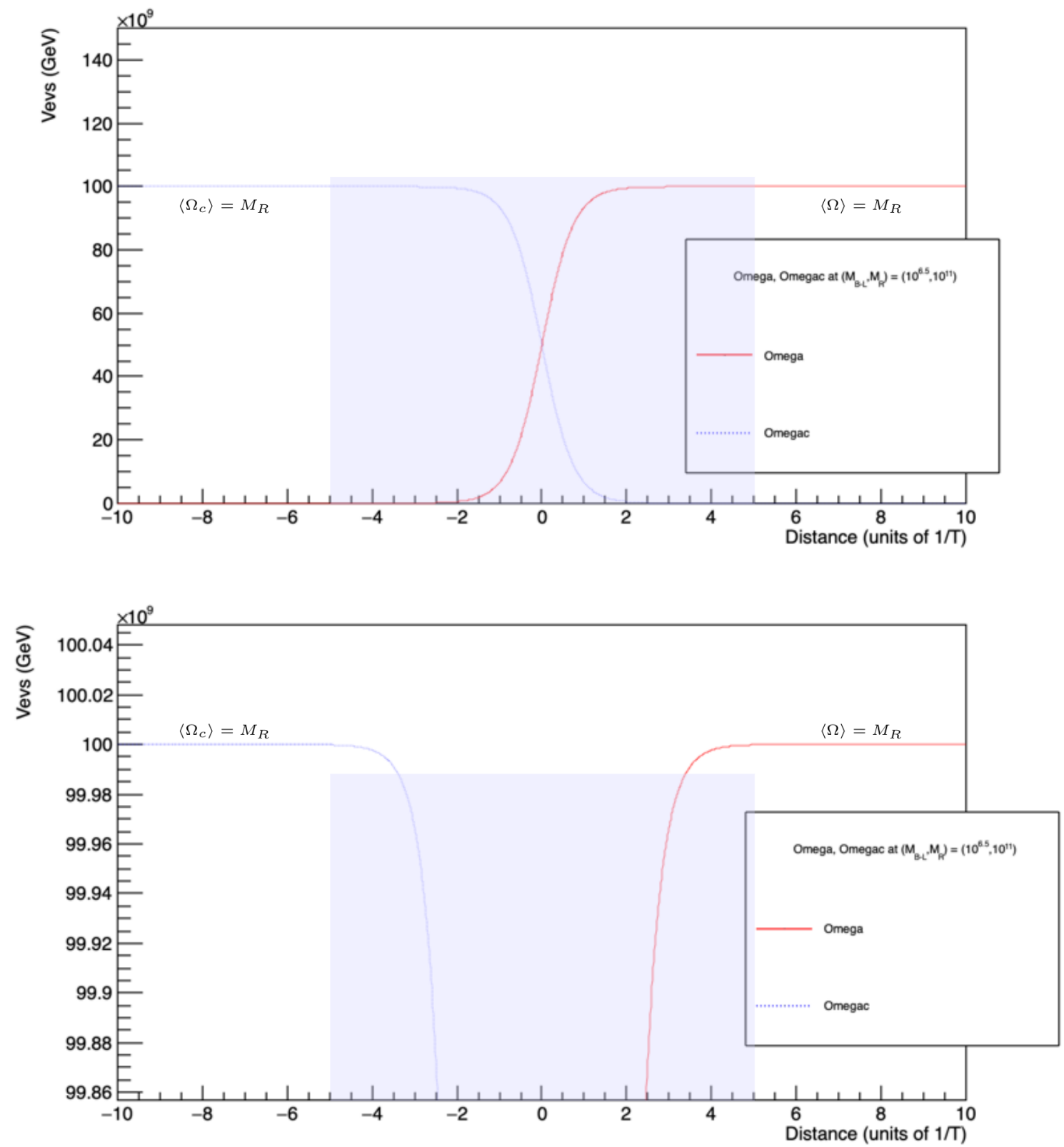

Figure 1. Above: plots of vacuum expectation values $\omega, \omega_{c}$ as a function of the distance $x$, in units of $1 / T$, from the domain wall, plotted for $T=M_{B-L}=10^{6.5} \mathrm{GeV}, M_{R}=10^{11} \mathrm{GeV}, L=5$, $\lambda \sim L^{-2}=0.04$ and the choice of the other parameters, $a=-1.5, \alpha=0.006, \mu_{11}=0.7 M_{R}$, $\mu_{22}=0.7 M_{R}, \mu_{1}=0.2 \alpha^{2} M_{R}, \mu_{2}=0.3 \alpha^{2} M_{R}, \mu_{3}=0.5 \alpha^{2} M_{R}, \beta_{3}=-0.5, \mu_{4}=1.5 \alpha^{2} M_{R}, \beta_{4}=1.2$, $\mu_{5}=0.1 \alpha^{2} M_{R}, \beta_{5}=-1.4$. The domain wall stretches from $-L$ to $L$. Below: the same plot magnfied, showing the gradual drop of the vevs of $\omega, \omega_{c}$ just inside the wall around $x= \pm L$. 


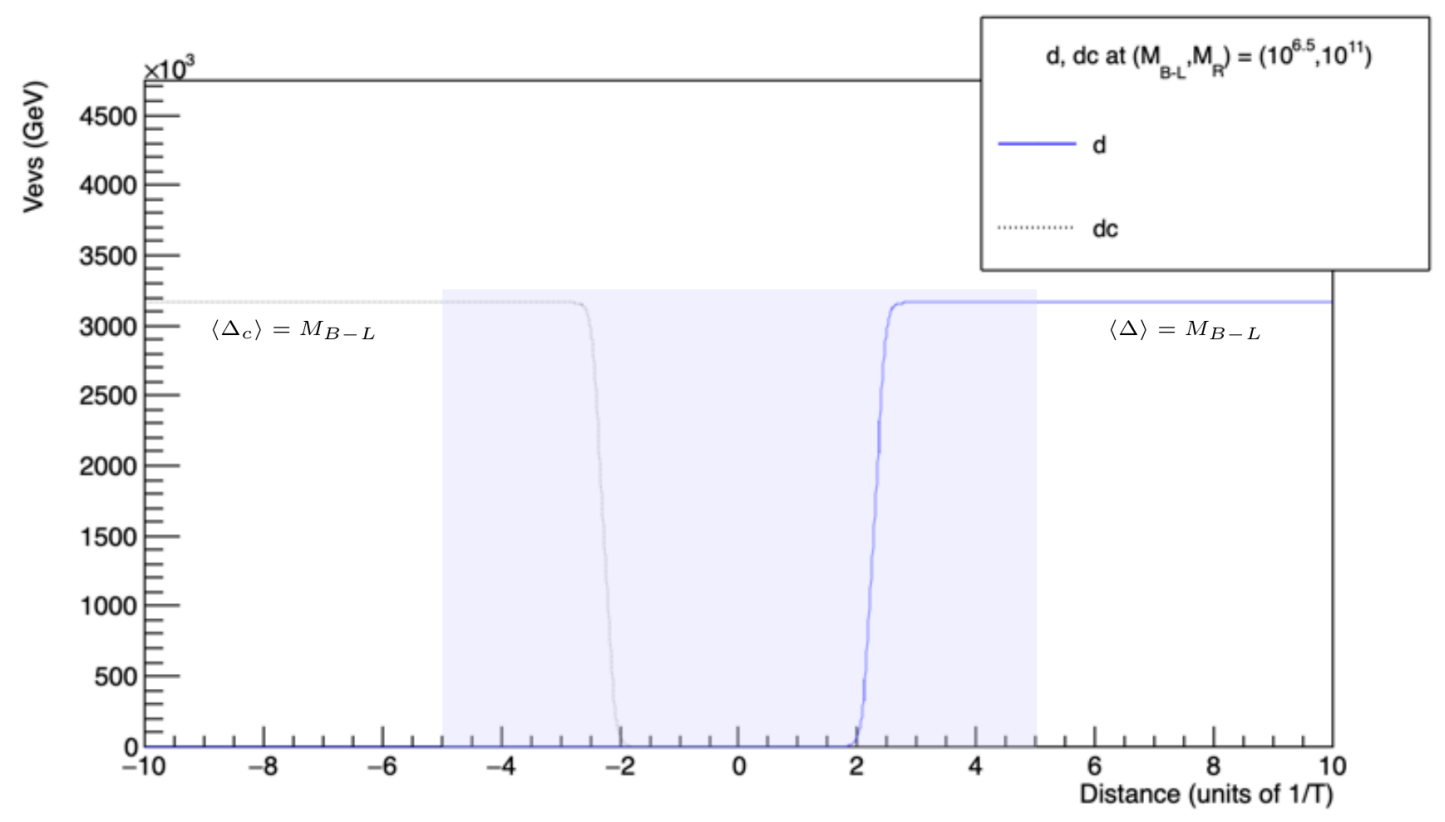

Figure 2. Plots of vacuum expectation values $d, d_{c}$ obtained for $T=M_{B-L}=10^{6.5} \mathrm{GeV}$, $M_{R}=10^{11} \mathrm{GeV}$, and for the ansatz and parameters as in figure 1.

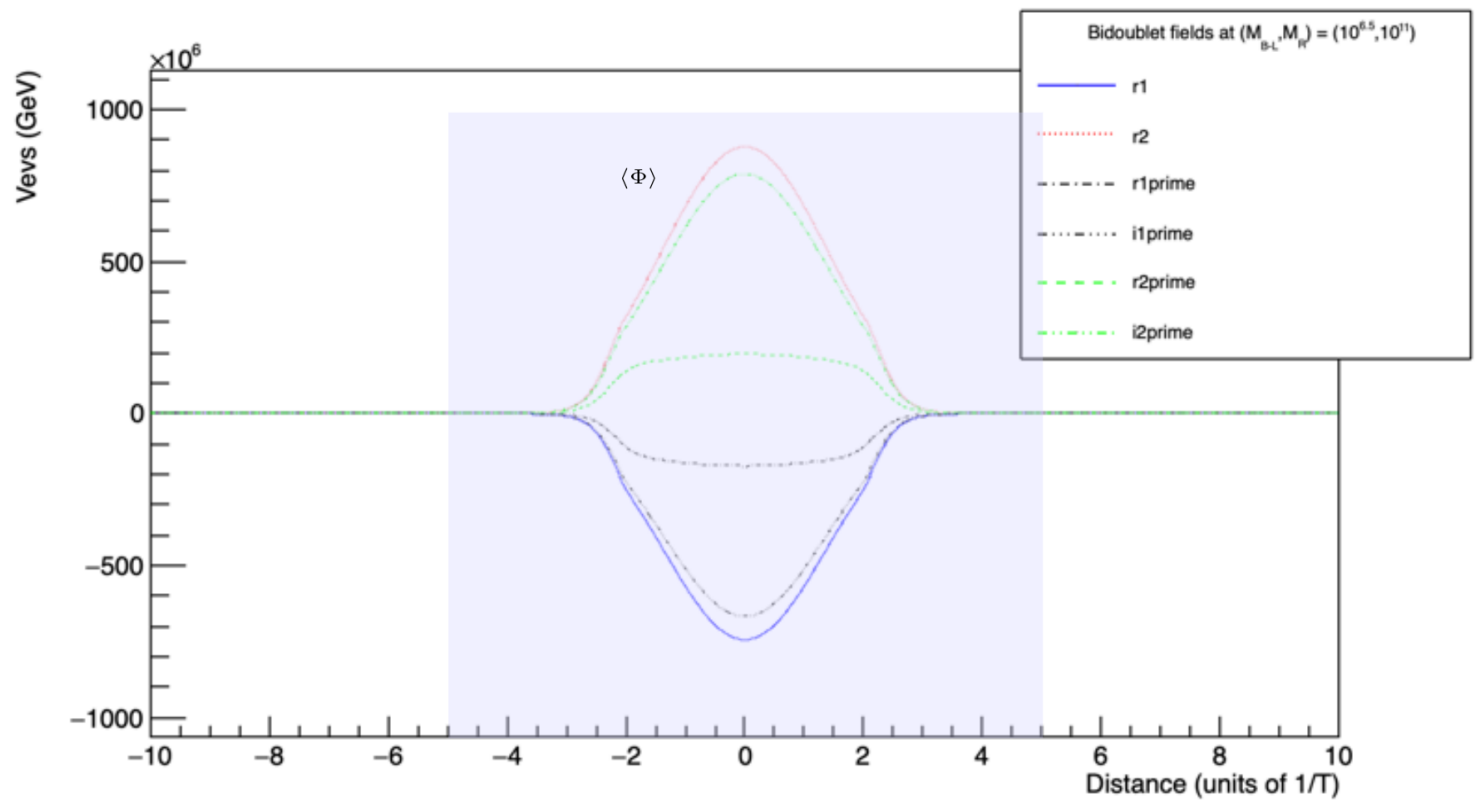

Figure 3. Real and imaginary parts of the bidoublet Higgs fields, $r_{1}, r_{1}^{\prime}, i_{1}^{\prime}, r_{2}, r_{2}^{\prime}, i_{2}^{\prime}$, as a function of the distance $x$, in units of $1 / T$, from the domain wall, plotted for $T=M_{B-L}=10^{6.5} \mathrm{GeV}$, $M_{R}=10^{11} \mathrm{GeV}$, and other parameters as in figure 1. 


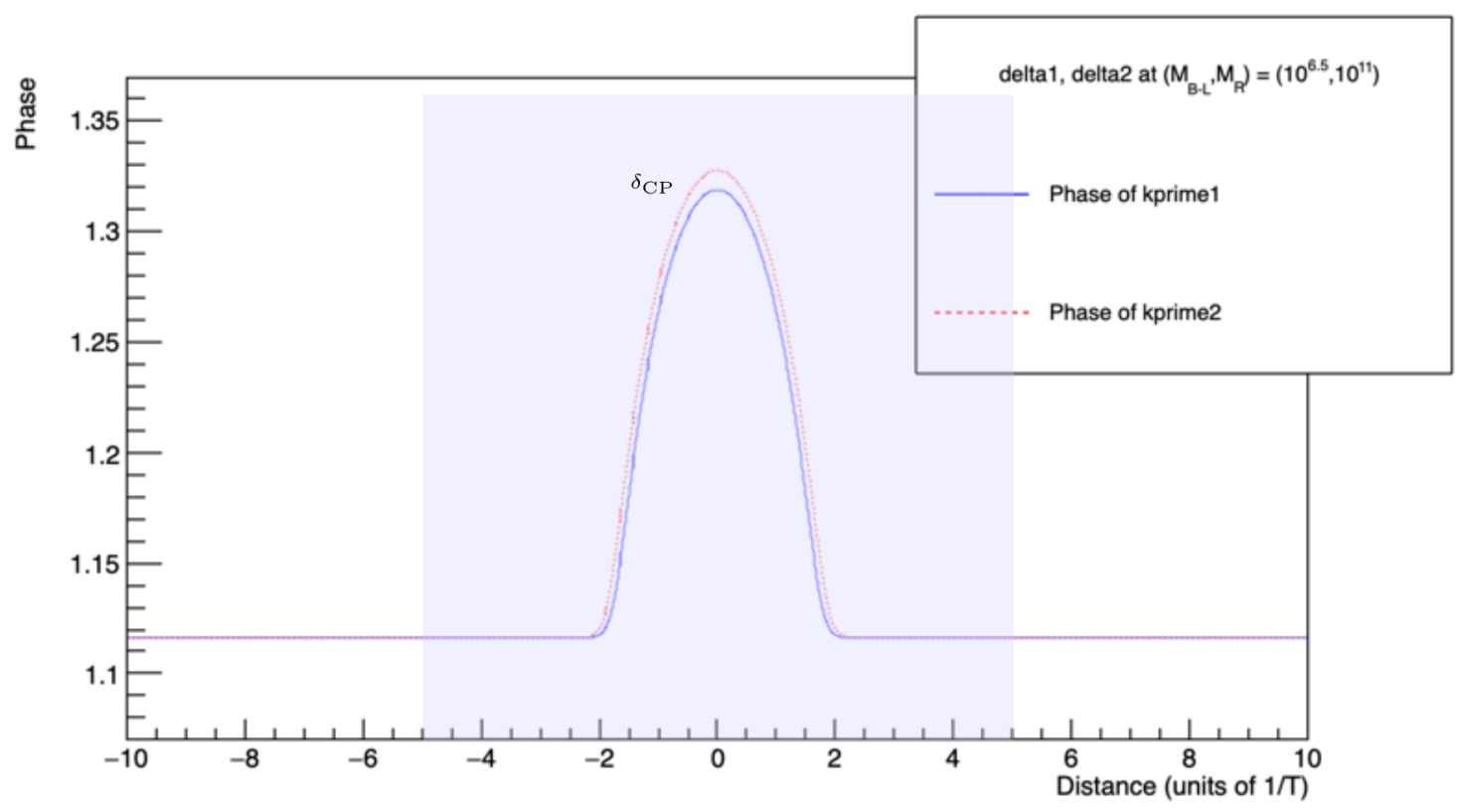

Figure 4. The phases of $k_{1}^{\prime}, k_{2}^{\prime}$ plotted as a function of $x$ for the same parameters as in figure 1 and figure 3 .

The one loop contribution to electron EDM $d_{e}$ is given by [35]

$$
\left.\left(d_{e} / e\right)\right|_{\text {one loop }} \sim \frac{\alpha m_{e}}{4 \pi M_{h}^{2}} \sin \delta,
$$

where $M_{h}$ is the mass of the lightest eigenstate of the bidoublet Higgs, $\alpha$ is the fine structure constant evaluated at the scale $M_{h}$ and $\delta$ is the complex relative phase between the neutral scalars induced by the lightest mass eigenstate. Surprisingly however, for large values of $M_{B-L}$ and $M_{R}$ two loop effects arising from the neutral scalars dominate the one loop effect. This was first realised by Barr and Zee [24] and then refined by several other authors. We use the formulas of Chang, Keung and Yuan [42] in order to compute the two loop contribution. The two loop contribution is a sum of contributions from several diagrams. Most important amongst those are four diagrams coming from figure 5 arising from the choice of top quark or $W$ boson in the inner loop, and the choice of $H \gamma \gamma$ or $H Z \gamma$ as the bosons interacting with this inner loop. Their total contribution is of the form

$$
\begin{aligned}
\left.\left(d_{e} / e\right)\right|_{\text {two loop }}=\frac{G_{F} m_{e} \alpha \sin \delta}{\pi^{3} \sqrt{2}} & \left(f_{W, H \gamma \gamma}\left(M_{W}^{2} / M_{h}^{2}\right)+f_{W, H Z \gamma}\left(M_{W}^{2} / M_{h}^{2}\right)\right. \\
& \left.+f_{t, H \gamma \gamma}\left(M_{t}^{2} / M_{h}^{2}\right)+f_{t, H Z \gamma}\left(M_{t}^{2} / M_{h}^{2}\right)\right) .
\end{aligned}
$$

where the functions $f$ are certain logarithmically growing functions defined in [24, 42].

We calculate the electron EDM numerically as a function of the LRSUSY model parameters $M_{B-L}$ and $M_{R}$ using ROOT version 6.16 libraries. We let $M_{B-L}$ range from $10^{4}$ to $10^{10} \mathrm{GeV}$. For a given $M_{B-L}$, we let $M_{R}$ range from a low of $10^{2} M_{B-L}$ to a high of $M_{B-L}^{2} / M_{\mathrm{EW}}$. The lower bound on $M_{R}$ allows us to safely break parity and left-right 


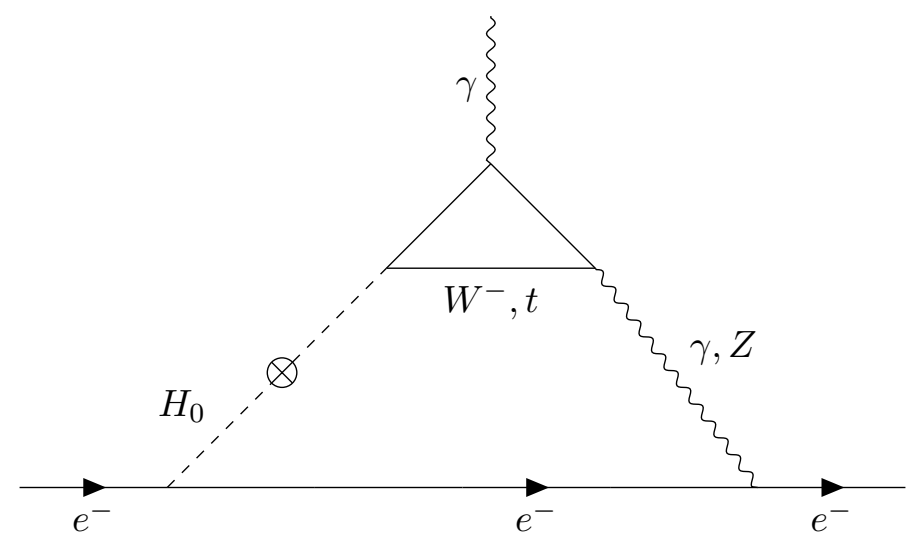

Figure 5. The two loop diagram giving the maximum contribution to the electron EDM in our model. Four such diagrams have to be calculated, corresponding to the choice of top quark or $W$ boson in the inner loop, and the choice of $H_{i} \gamma \gamma$ or $H_{i} Z \gamma$ as the bosons interacting with this inner loop. Here $H_{0}$ denotes the lightest mass eigenstate of the bidoublet Higgs.

symmetry before breaking R-parity, and the upper bound on $M_{R}$ allows the $\Omega$ fields in the left handed domain, which have a mass of about $M_{B-L}^{2} / M_{R}$, to stay heavier than the electroweak scale or the supersymmetry breaking scale [12]. The lower bound on $M_{B-L}$ ensures that it is above any reasonable supersymmetry breaking scale $M_{S}$ and so one can comfortably break the $\mathrm{U}(1)_{B-L}$ gauge symmetry to reduce to the MSSM. In other words, as argued in more detail in [12], the low energy effective theory of LRSUSY turns out to be the MSSM with strictly unbroken R-parity, and so the lightest supersymmetric particle is stable. The upper bound on $M_{B-L}$ follows from the consideration that for $M_{B-L} \geq 10^{10} \mathrm{GeV}$ we have to take $M_{R} \geq 10^{12} \mathrm{GeV}$ which is rather high for parity breaking. The experimentally allowed region, where the electron EDM, is less than $1.1 \times 10^{-29} \mathrm{e} \mathrm{cm} \mathrm{[19],} \mathrm{is} \mathrm{plotted}$ as the green hatched region in the $\left(M_{B-L}, M_{R}\right)$-plane in figure 6 .

\section{Leptogenesis constraints on LRSUSY}

The non-supersymmetric LR model was studied in the context of conventional electroweak baryogenesis mechanisms in $[43,44]$, and in a domain wall mediated baryogenesis via leptogenesis mechanism in [15]. The possibility of extending the latter mechanism to LRSUSY was indicated in [16], but no concrete numerical calculations were performed there. In this paper, we address this deficiency.

Adequate amount of $\mathrm{CP}$ violation as well as strong loss of equilibrium conditions have been major challenges for low energy baryogenesis. The presence of a moving domain wall, a topological defect, towards the energetically disfavoured right handed domain immediately guarantees a strong loss of equilibrium. The main LRSUSY model per se does not explain why this happens, but we assume that this occurs because of tiny effects like soft SUSY terms [16] or Planck suppressed non-renormalisable terms [31] breaking exact leftright symmetry. A similar calculation has recently been done [45] showing how Planck suppressed non-renormalisable terms can remove a pseudo domain wall in supersymmetric 
$\mathrm{SO}(10)$ GUT without conflicting with standard cosmology. Given that the domain wall in LRSUSY has somehow disappeared early enough so as not to conflict with present day cosmology, we can exploit it to obtain leptogenesis and consequent baryogenesis consistent with experimental bounds on baryon asymmetry. This is done as follows.

The lepton-Higgs Yukawa part of the superpotential of LRSUSY is [12]

$$
W_{Y}=\mathbf{h}_{l}^{(j)} L^{T} \tau_{2} \Phi_{j} \tau_{2} L_{c}+i \mathbf{f}\left(L^{T} \tau_{2} \Delta L+L_{c}^{T} \tau_{2} \Delta_{c} L_{c}\right),
$$

where $j=1,2$ and $\mathbf{h}, \mathbf{f}$ are $3 \times 3$ real symmetric matrices. The Majorana mass terms above corresponding to the Yukawa coupling matrix $\mathbf{f}$ are a source of lepton number violation. However, in LRSUSY they do not favour conventional thermal leptogenesis because at the usual scale of thermal leptogenesis, the $B-L$ gauged symmetry is unbroken [16]. That is why we have to resort to domain wall mediated leptogenesis in LRSUSY. The lepton number violating decay of the heavy Majorana RH neutrino will instead give rise to a lepton asymmetry washout which will dilute any lepton asymmetry mediated by the domain wall. Nevertheless, as we will see below, it is possible to obtain baryon asymmetry consistent with experimental data for a certain region of the LRSUSY parameter space.

Consider a domain wall moving slowly with speed $v_{w}$ in the $+x$ direction i.e. encroaching upon the energetically disfavoured $\mathrm{RH}$ domain. The wall is assumed to stretch from $-L$ to $+L$ in the $x$ direction and be flat in the $y z$ plane. Slow speed means that $v_{w}<1 / \sqrt{3}$, the speed of sound in the hot plasma, allowing one to get a solution to the chemical potential of the neutrinos in terms of a fluid approximation [46]. Since the wall is assumed to move due to tiny energy differences between the $\mathrm{LH}$ and $\mathrm{RH}$ domain, this is a reasonable assumption. We consider the case of thick walls i.e. $2 L>1 / T$, the de Broglie wavelength of the neutrinos at temperature $T$ [46]. This is a reasonable assumption ensuring that the mean free path of the neutrinos is smaller than the wall thickness, leading to multiple interactions between neutrinos and the $\mathrm{CP}$ violating condensate in the wall and allowing a classical WKB treatment of the neutrinos. A wall thickness of $10 / T$ will be typical in our analysis. We assume that the neutrino diffusion coefficient $D<2 L /\left(3 v_{w}\right)$ [46], allowing us to do a thermalised fluid analysis of the chemical potential of the LH neutrinos. The expression for $D$ below easily satisfies this constraint.

Consider the interaction of LH neutrinos with the domain wall. The LH neutrinos $\nu_{L}$ are massive in the $\mathrm{RH}$ domain due to the Majorana Yukawa coupling to the $\mathrm{SU}(2)_{L^{-}}$triplet Higgs field $\Delta$ which takes a vev in the $\mathrm{RH}$ domain. Conversely they are massless in the LH domain where $\Delta$ has a vanishing vev. Due to the existence of a spatially varying CP violating condenstate arising from the complex bidoubet vevs inside the wall, one get an asymmetry between $\nu_{L}$ and its antiparticle $\bar{\nu}_{L}$ in terms of their reflection and transmission coefficients with respect to the wall. There will be a preference for transmission of, say, $\nu_{L}$ from $\mathrm{RH}$ domain to $\mathrm{LH}$ domain through the wall. The LH neutrinos reflected back into $\mathrm{RH}$ domain from the wall quickly equilibrate with their antiparticles around them because of the high rate of helicity flipping of LH neutrinos owing to their large Majorana mass in the $\mathrm{RH}$ domain. Thus the $\mathrm{RH}$ domain continues to have almost no particle antiparticle asymmetry. In contrast, the transmitted excess of LH neutrinos into LH domain survives because they are almost massless in LH domain and hardly flip helicity. This leads to a 
particle antiparticle asymmetry in LH domain. Eventually as the domain wall encroaches totally upon $\mathrm{RH}$ domain and destroys it, we end up with an excess of leptons in our left handed Universe. Weak sphaeleron processes convert a part of the early lepton excess into a baryon excess till they go out of equilibrium as our left handed Universe cools. Thus, one is left with a baryon excess in the present day Universe.

The diffusion equation for the chemical potential $\mu$ of the LH neutrino in the wall rest frame is given by [15]

$$
-D \mu^{\prime \prime}+v_{w} \mu^{\prime}+\Gamma \Theta(x) \mu=S(x),
$$

where $S(x)$ is the so-called source term defined below, $D$ is the neutrino diffusion coefficient, $v_{w}$ is the speed of the wall taken to be moving in the $+x$ direction, $\Theta(x)$ is the step function which takes value one if $x$ is positive and zero otherwise, $\Gamma$ is the rate of helicity flipping interactions at temperature $T$ which is very high in the $\mathrm{RH}$ domain due to the heavy Majorana mass of the $\mathrm{LH}$ neutrino in the $\mathrm{RH}$ domain and zero in the $\mathrm{LH}$ domain since the LH neutrino is almost massless in the LH domain. Observe that the LH neutrino mass $m_{\nu}(x)$ is spatially dependent and complex inside the wall since the neutrino couples to the spatially varying complex bidoublet Higgs and the triplet Higgs fields $\Delta, \Delta_{c}$ quickly vanish inside the wall (see figures 2,3 ).

The source term, which is a CP-violating non-zero force if the neutrino mass $m_{\nu}(x)$ and the domain wall CP phase $\delta(x)$ are spatially varying, is given by [47]

$$
S(x)=-\frac{v_{w} D}{2 \Gamma}\left\langle\frac{\left|p_{x}\right|}{E^{2} \tilde{E}}\right\rangle\left(m_{\nu}(x) \delta^{\prime}(x)\right)^{\prime \prime},
$$

where $p_{x}$ is the $x$-component of the LH neutrino's momentum, $E$ is the neutrino energy, $\tilde{E}$ is the related quantity $\sqrt{m_{\nu}(x)^{2}+p_{x}^{2}}$, and the angular brackets indicate thermal averages. It was shown in [47] that

$$
\left\langle\frac{\left|p_{x}\right|}{E^{2} \tilde{E}}\right\rangle=\frac{e^{-a}-a E_{1}(a)}{2 T^{2} a^{2} K_{2}(a)},
$$

where $a=m_{\nu}(\infty) / T, E_{1}$ is the error function, $K_{2}$ is the modified cylindrical Bessel function of the second kind and the LH neutrino mass is evaluated deep inside the RH domain. The source term is zero outside the wall but non-zero inside.

The helicity flipping rate can be calculated by [46]

$$
\Gamma=\frac{\alpha_{w}^{2} m_{\nu}(\infty)^{2}}{T},
$$

where $\alpha_{w}$ is the weak coupling constant evaluated at temperature $T$. The diffusion coefficient has the expression [47],

$$
D=\frac{\left\langle v_{x}^{2}\right\rangle}{\Gamma}, \quad\left\langle v_{x}^{2}\right\rangle=\frac{3 a+2}{a^{2}+3 a+2},
$$

where $v_{x}$ is the $x$-component of the neutrino velocity and $a$ is defined above.

We solve the diffusion equation (equation (6.2)) numerically the using GSL version 2.6 library under the same settings as before, and take the limiting value $\mu(-\infty)$ in the $\mathrm{LH}$ 
domain as the steady state chemical potential of the LH neutrino. Then, the steady state neutrino-antineutrino asymmetry in the LH domain becomes [15]

$$
\Delta_{\nu_{L}}=\frac{T^{2}}{6} \mu(-\infty)
$$

To obtain the raw lepton asymmetry to entropy density ratio $\eta^{\text {raw }}$, we need to divide the above quantity by $\frac{2 \pi^{2} g_{*} T^{3}}{45}$, where $g_{*} \approx 110$ is the number of relativistic degrees of freedom. Doing so gives us

$$
\eta_{L}^{\mathrm{raw}}=0.0035 \frac{\mu(-\infty)}{T} .
$$

Since the heavy neutrinos in this model have mass less than the temperature $M_{B-L}$, they can easily decay violating lepton number. This process washes out most of the raw lepton asymmetry $\eta_{L}^{\text {raw }}$. The surviving lepton asymmetry by entropy density ratio becomes [15]

$$
\eta_{L}=\eta^{\mathrm{raw}} \cdot 10^{-4 \cdot 10^{-4} m_{\nu} M_{\mathrm{Planck}} v^{-2}}=3.054 \cdot 10^{-11} \frac{\mu(-\infty)}{T}
$$

where $m_{\nu}$ is the mass of the heaviest light neutrino and $v=174 \mathrm{GeV}$ is the SM Higgs vev. We take $m_{\nu}=0.05 \mathrm{eV}$ from the Nu-FIT Group [48].

Finally electroweak sphaelerons convert part of the lepton asymmetry to baryon asymmetry starting from the temperature $M_{B-L}$ till the universe cools to the sphaeleron scale of about one $\mathrm{TeV}$. This gives the steady state baryon asymmetry to entropy density ratio [15]

$$
\eta_{B}=\frac{28}{51} \eta_{L}=1.677 \cdot 10^{-11} \frac{\mu(-\infty)}{T} .
$$

The yellow strip in figure 6 shows the $\left(M_{R}, M_{B-L}\right)$ tuples where the final baryon asymmetry to entropy density ratio is between $10^{-11}$ and $10^{-8}$, which can thus provide a good explanation of the experimentally observed baryon asymmetry of $6 \cdot 10^{-10}[17]$. The parameter space of the yellow strip is consistent with the experimental upper bound on electron EDM indicated by the green hatched region in figure 6 .

In ending this section we compare our low scale leptogenesis scenario with the more popular versions of electroweak scale baryogenesis. In the vanilla Standard Model, a first order phase transition is not possible during EW symmetry breaking because the Higgs mass is well above $80 \mathrm{GeV}$. Moreover, the $\mathrm{CP}$ violation from the CKM matrix in $\mathrm{SM}$ is insufficient to explain the observed baryon asymmetry of the universe. It is possible to ensure first order phase transition by adding more Higgs doublets or singlets to the electroweak sector. For example, in the MSSM the lightest stop can enhance the first order EW phase transition. However there are other phenomenological constraints to be met, and it turns out that a first order phase transition is only marginally consistent with MSSM [35]. Other multi Higgs models proposed to enhance the phase transition require new physics at the $\mathrm{TeV}$ scale. In the absence of any such evidence from recent LHC data, the case for these extended electroweak models to enhance the phase transition becomes weakened.

In contrast, for the LRSUSY model considered in this paper which is well motivated by other theoretical considerations and is consistent with the latest direct upper bounds on the neutrino mass [49], we show that baryogenesis via domain wall mediated leptogenesis is 


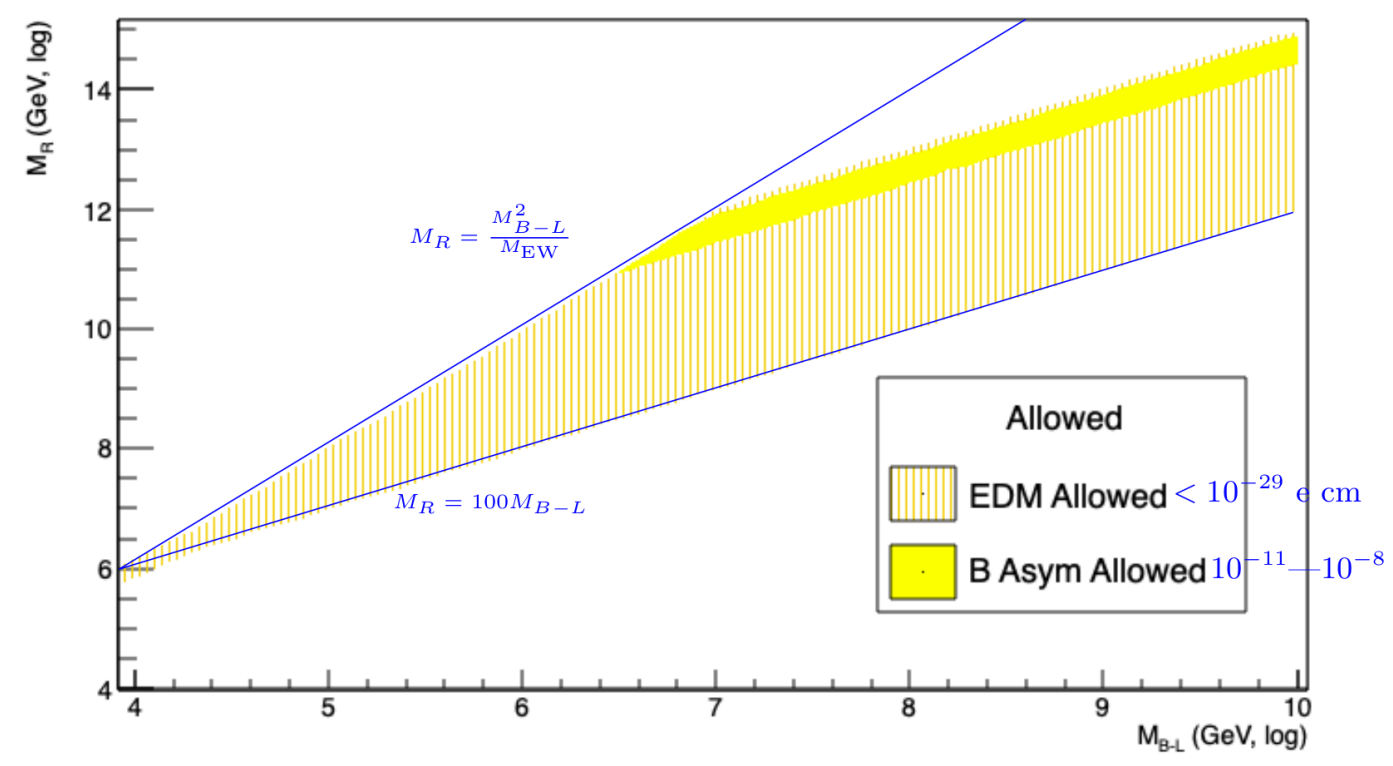

Figure 6. The regions in the $\left(M_{B-L}, M_{R}\right)$-plane allowed by the experimental bounds on baryon asymmetry in yellow and electron EDM hatched green, plotted for the same parameter set as figure 1-4. The upper blue line denotes the setting $M_{R}=M_{B_{L}}^{2} / M_{E W}$ and the lower blue line $M_{R}=100 M_{B-L}$. The baryon asymmetry allowed region is the narrow region entirely subsumed within that allowed by the EDM constraint and overlaid on the latter. The two regions run parallel at upper boundary with EDM allowed region somewhat larger. To be specific, $10^{6.5}<M_{B-L}<$ $10^{10} \mathrm{GeV}$ and bears out the philosophy of eq. (2.14) only towards the lowest end of the range.

ubiquitous enough so as to explain the observed baryon asymmetry of the present universe. The domain walls automatically satisfy the requirement of a first order phase transition. We refer to earlier works to justify their disappearance early enough before electroweak symmetry breaking. We have shown that even these short lived domain walls can produce the right amount of baryon asymmetry for a large region of parameter space while staying consistent with electron EDM bounds.

\section{$7 \quad$ Primordial gravitational wave signature}

While we have been able to correlate two important signatures arising from this model an independent one can be identified due to the inhomogeneous behaviour of the domain walls. Gravitational waves are indeed produced by the annhilation of the domain walls that were originally formed by the breaking of the discrete parity symmetry. Previous works have studied gravitational waves arising from the breaking of bubble walls in general first order phase transitions above electroweak scale [50,51], breaking of bubble walls in electroweak baryogenesis in the non-SUSY LRSM model $[52,53]$, or from the breaking of domain walls in the non-SUSY two Higgs doublet model [25]. In this section, we do a quick study of gravitational waves arising from the breaking of domain walls in LRSUSY. We assume that the annihilation takes place at a temperature $T_{\text {ann }}$ below the supersymmetry breaking scale $M_{S}$. Hence the number of relativistic degrees of freedom at temperature $T_{\text {ann }}$ is the 
same as it would be in the Standard Model. The peak frequency of these waves is given by $[54]$

$$
f_{\text {peak }}=1.1 \times 10^{-7} \mathrm{~Hz}\left(\frac{g_{*}\left(T_{\mathrm{ann}}\right)}{10}\right)^{1 / 2}\left(\frac{g_{* s}\left(T_{\mathrm{ann}}\right)}{10}\right)^{-1 / 3}\left(\frac{T_{\mathrm{ann}}}{\mathrm{GeV}}\right),
$$

where $g_{*}\left(T_{\mathrm{ann}}\right), g_{* s}\left(T_{\mathrm{ann}}\right)$ are the number of relativistic degrees of freedom for the energy density and entropy density respectively at temperature $T_{\text {ann }}$. The peak energy density spectrum at the present time $t_{0}$ is given by [54]

$$
\Omega_{\mathrm{gw}} h^{2}\left(t_{0}\right)_{\mathrm{peak}}=2.02 \times 10^{-43}\left(\frac{g_{*}\left(T_{\mathrm{ann}}\right)}{10}\right)^{-4 / 3}\left(\frac{\sigma}{\mathrm{GeV}^{3}}\right)^{2}\left(\frac{T_{\mathrm{ann}}}{\mathrm{GeV}}\right)^{-4},
$$

where $\sigma$ is the surface energy density of the domain wall. Since the wall forms at temperature $M_{R}$, its surface energy density is well approximated by $\sigma \sim M_{R}^{3}$. Let us consider an intermediate scale of $M_{R}=10^{9} \mathrm{GeV}$ and annhilation temperature $T_{\text {ann }}=200 \mathrm{GeV}$ just above the electroweak scale and well below the SUSY breaking scale. At this annhilation temperature both $g_{*}\left(T_{\text {ann }}\right)$ and $g_{* s}\left(T_{\text {ann }}\right)$ are around 105 . The peak frequency and energy density of gravitational waves is now

$$
\begin{aligned}
f_{\text {peak }}(200 \mathrm{GeV}) & =3.256 \times 10^{-5} \mathrm{~Hz} \\
\Omega_{\mathrm{gw}} h^{2}\left(t_{0}\right)_{\text {peak }}\left(10^{9} \mathrm{GeV}\right) & =5.5 \times 10^{-54}\left(\frac{M_{R}}{\mathrm{GeV}}\right)^{6}=5.5 .
\end{aligned}
$$

This frequency is about six orders of magnitude below what LIGO [55] can detect today. However, proposed space-based gravitational wave detectors like eLISA [56] may be able to detect such frequencies and energy densities, serving as an important test of the model.

\section{Discussion and conclusion}

Armed with our numerical calculation tools, we have made a strategic exploration of the parameter space of LRSUSY to find the subregion consistent with the current experimental bounds on electron EDM and baryon-antibaryon asymmetry. We have varied the trilinear $\Omega \Phi \Phi$ coupling parameter $\alpha$ from 0.001 to 0.1 , and the mass parameters $\mu$ from 0.5 to 1 in order to study the shape of the Higgs fields inside the wall and have obtained similar results as described above. These ranges of the parameters were chosen from the following considerations. Values of $\alpha$ greater than 0.1 make the soft terms not so soft anymore, and values of mass parameters $\mu$ outside the above range either make the bidoublets heavier than the largest mass scale $M_{R}$ or make our fine tuning ineffective. The manifestation of the limiting values of the parameters shows up in the observation that for $\mu_{11}, \mu_{22}$ smaller than $0.5 M_{R}$, or for $\alpha>0.1$, there is no value of $M_{B-L}$ between $10^{4}$ to $10^{10} \mathrm{GeV}$ consistent with both electron EDM and baryon asymmetry experiments.

For parameter values in the above ranges, we see that the lowest $M_{B-L}$ consistent with the two experiments is $10^{4.5} \mathrm{GeV}$. The lowest allowed value of $M_{B-L}$ is most sensitive to $\alpha$, and is a decreasing function of $\alpha$. Figure 7 shows its variation when $\alpha$ ranges from 0.001 to 0.1 . 


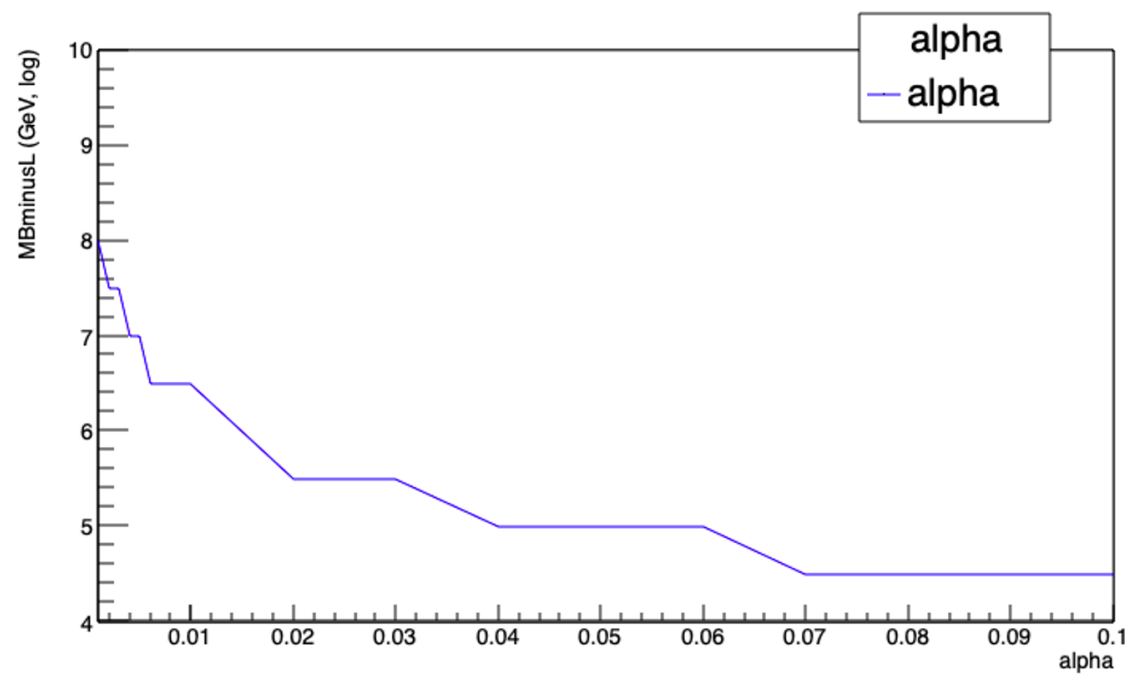

Figure 7. The lowest value of $M_{B-L}$ consistent with both electron EDM and baryon asymmetry experiments plotted as a function of $\alpha$.

It turns out that the lowest allowed value of $M_{B-L}$ is hardly sensitive to the mass parameters and variations in wall thickness and wall velocity. Thus, by scanning the parameter space we can conclude that for consistency with both electron EDM and baryon asymmetry experiments, the scale $M_{B-L}$ of $B-L$-symmetry breaking must be larger than $10^{4.5} \mathrm{GeV}$, and over the whole allowed range must be significantly less that $M_{B-L}^{2} / M_{E W}$. These novel bounds provide the most stringent constraints on the parameter space of LRSUSY by far.

More interestingly, our implication that $M_{R}$ must be significantly less than $M_{B-L}^{2} / M_{E W}$ accords with the simplifying proposal of Aulakh et al. [12] viz. $M_{R} \gtrsim M_{B-L}^{2} / M_{E W}$, as discussed at (2.14), only at the lowest allowed value of $M_{B-L}$. Aulakh et al.'s proposal arose from gravity mediated TeV scale SUSY breaking where the gravitino is not much heavier than $M_{E W}$. Within the validity of this proposal this analysis makes a specific prediction of the $M_{B-L}$ and hence the $M_{R}$. Since LHC has not discovered any signatures of SUSY, TeV scale SUSY breaking is now a highly unlikely possibility though an exciting one to confirm if true.

On the other hand we can reject the $R$ parity proposed in [12], in order to allow the fact that the scale of parity breaking and $(B-L)$-symmetry breaking cannot be too far apart. The parameter $m_{\Omega}$ could then be intrinsic to the superpotential. In this case our results are perfectly consistent with $\mathrm{PeV}$ scale supersymmetry [57] also considered in the recent work [58], where the gravitino can be much heavier. This places LRSUSY outside the experimental reach of colliders in the near future. On the other hand, the discovery of a non-zero electron EDM value can be taken to be a hint to a narrow range for the $M_{R}$ scale assuming a world with a renormalisable supersymmetric left-right model. Finally it is interesting that two very different low-energy probes viz. baryon asymmetry and electron EDM provide a strong constraint on the allowed parameter space of LRSUSY. 


\section{A Euler-Lagrange equations for spatially varying Higgs vevs}

The Euler-Lagrange equations are written explicitly below, where $\ddot{f}$ represents the double derivative of vev of field $f$ with respect to $x$ and $F_{\left(\Phi_{i}\right)_{j}}$ denotes the $(j, j)$ matrix element of the vev $\left\langle F_{\Phi_{i}}\right\rangle$. The expressions for the vevs of the F-terms and D-terms can be found in equations (2.8) and (2.6) respectively. There are eight bidoublet field vevs $r_{1}, i_{1}, \ldots, r_{2}^{\prime}, i_{2}^{\prime}$ which are the real and imaginary parts of the vev $k_{1}, \ldots, k_{2}^{\prime}$ respectively. There are 4 triplet fields vevs viz. $d, \bar{d}, d_{c}, \bar{d}_{c}$. All these vevs vary as a function of distance $x$ from the centre of the wall, but we write field $f$ in the equations below and not $f(x)$ for clarity of notation. The vevs of the fields $\omega(x), \omega_{c}(x)$ below take their values from the ansatz in equation (4.1).

$$
\begin{aligned}
& \ddot{r}_{1}=4 \alpha \operatorname{Re}\left(F_{\Omega}+F_{\Omega_{c}}\right) r_{2}^{\prime}+8 \alpha \operatorname{Im}\left(F_{\Omega}\right) i_{2}^{\prime}+8 \mu_{11} \operatorname{Re}\left(F_{\left(\Phi_{1}\right)_{2}}\right) \\
& +8\left(\mu_{12}+\alpha\left(\omega-\omega_{c}\right)\right) \operatorname{Re}\left(F_{\left(\Phi_{2}\right)_{2}}\right)+8 g^{2} r_{1} D_{L, 3} \\
& -2 \mu_{1}^{2} r_{1}-4 \mu_{3}^{2}\left(r_{1}^{\prime} \cos \beta_{3}+i_{1}^{\prime} \sin \beta_{3}\right)-2 \mu_{5}^{2}\left(r_{2}^{\prime} \cos \beta_{5}+i_{2}^{\prime} \sin \beta_{5}\right), \\
& \ddot{i}_{1}=-4 \alpha \operatorname{Re}\left(F_{\Omega}+F_{\Omega_{c}}\right) i_{2}^{\prime}+8 \alpha \operatorname{Im}\left(F_{\Omega}\right) r_{2}^{\prime}+8 \mu_{11} \operatorname{Im}\left(F_{\left(\Phi_{1}\right)_{2}}\right) \\
& +8\left(\mu_{12}+\alpha\left(\omega-\omega_{c}\right)\right) \operatorname{Im}\left(F_{\left(\Phi_{2}\right)_{2}}\right)+8 g^{2} i_{1} D_{L, 3} \\
& -2 \mu_{1}^{2} i_{1}-4 \mu_{3}^{2}\left(-i_{1}^{\prime} \cos \beta_{3}+r_{1}^{\prime} \sin \beta_{3}\right)-2 \mu_{5}^{2}\left(-i_{2}^{\prime} \cos \beta_{5}+r_{2}^{\prime} \sin \beta_{5}\right) \text {, } \\
& \ddot{r}_{2}=-4 \alpha \operatorname{Re}\left(F_{\Omega}+F_{\Omega_{c}}\right) r_{1}^{\prime}-8 \alpha \operatorname{Im}\left(F_{\Omega}\right) i_{1}^{\prime}+8 \mu_{22} \operatorname{Re}\left(F_{\left(\Phi_{2}\right)_{2}}\right) \\
& +8\left(\mu_{12}-\alpha\left(\omega-\omega_{c}\right)\right) \operatorname{Re}\left(F_{\left(\Phi_{1}\right)_{2}}\right)+8 g^{2} r_{2} D_{L, 3} \\
& -2 \mu_{2}^{2} r_{2}-4 \mu_{4}^{2}\left(r_{2}^{\prime} \cos \beta_{4}+i_{2}^{\prime} \sin \beta_{4}\right)-2 \mu_{5}^{2}\left(r_{1}^{\prime} \cos \beta_{5}+i_{1}^{\prime} \sin \beta_{5}\right), \\
& \ddot{i}_{2}=4 \alpha \operatorname{Re}\left(F_{\Omega}+F_{\Omega_{c}}\right) i_{1}^{\prime}-8 \alpha \operatorname{Im}\left(F_{\Omega}\right) r_{1}^{\prime}+8 \mu_{22} \operatorname{Im}\left(F_{\left(\Phi_{2}\right)_{2}}\right) \\
& +8\left(\mu_{12}-\alpha\left(\omega-\omega_{c}\right)\right) \operatorname{Im}\left(F_{\left(\Phi_{1}\right)_{2}}\right)+8 g^{2} i_{2} D_{L, 3} \\
& -2 \mu_{2}^{2} i_{2}-4 \mu_{4}^{2}\left(-i_{2}^{\prime} \cos \beta_{4}+r_{2}^{\prime} \sin \beta_{4}\right)-2 \mu_{5}^{2}\left(-i_{1}^{\prime} \cos \beta_{5}+r_{1}^{\prime} \sin \beta_{5}\right) \text {, } \\
& \ddot{r}_{1}^{\prime}=-4 \alpha \operatorname{Re}\left(F_{\Omega}+F_{\Omega_{c}}\right) r_{2}-8 \alpha \operatorname{Im}\left(F_{\Omega}\right) i_{2}+8 \mu_{11} \operatorname{Re}\left(F_{\left(\Phi_{1}\right)_{1}}\right) \\
& +8\left(\mu_{12}-\alpha\left(\omega-\omega_{c}\right)\right) \operatorname{Re}\left(F_{\left(\Phi_{2}\right)_{1}}\right)-8 g^{2} r_{1}^{\prime} D_{L, 3} \\
& -2 \mu_{1}^{2} r_{1}^{\prime}-4 \mu_{3}^{2}\left(r_{1} \cos \beta_{3}+i_{1} \sin \beta_{3}\right)-2 \mu_{5}^{2}\left(r_{2} \cos \beta_{5}+i_{2} \sin \beta_{5}\right), \\
& \ddot{i}_{1}^{\prime}=4 \alpha \operatorname{Re}\left(F_{\Omega}+F_{\Omega_{c}}\right) i_{2}-8 \alpha \operatorname{Im}\left(F_{\Omega}\right) r_{2}+8 \mu_{11} \operatorname{Im}\left(F_{\left(\Phi_{1}\right)_{1}}\right) \\
& +8\left(\mu_{12}-\alpha\left(\omega-\omega_{c}\right)\right) \operatorname{Im}\left(F_{\left(\Phi_{2}\right)_{1}}\right)-8 g^{2} i_{1}^{\prime} D_{L, 3} \\
& -2 \mu_{1}^{2} i_{1}^{\prime}-4 \mu_{3}^{2}\left(-i_{1} \cos \beta_{3}+r_{1} \sin \beta_{3}\right)-2 \mu_{5}^{2}\left(-i_{2} \cos \beta_{5}+r_{2} \sin \beta_{5}\right), \\
& \ddot{r}_{2}^{\prime}=4 \alpha \operatorname{Re}\left(F_{\Omega}+F_{\Omega_{c}}\right) r_{1}+8 \alpha \operatorname{Im}\left(F_{\Omega}\right) i_{1}+8 \mu_{22} \operatorname{Re}\left(F_{\left(\Phi_{2}\right)_{1}}\right) \\
& +8\left(\mu_{12}+\alpha\left(\omega-\omega_{c}\right)\right) \operatorname{Re}\left(F_{\left(\Phi_{1}\right)_{1}}\right)-8 g^{2} r_{2}^{\prime} D_{L, 3} \\
& -2 \mu_{2}^{2} r_{2}^{\prime}-4 \mu_{4}^{2}\left(r_{2} \cos \beta_{4}+i_{2} \sin \beta_{4}\right)-2 \mu_{5}^{2}\left(r_{1} \cos \beta_{5}+i_{1} \sin \beta_{5}\right) \text {, } \\
& \ddot{i}_{2}^{\prime}=-4 \alpha \operatorname{Re}\left(F_{\Omega}+F_{\Omega_{c}}\right) i_{1}+8 \alpha \operatorname{Im}\left(F_{\Omega}\right) r_{1}+8 \mu_{22} \operatorname{Im}\left(F_{\left(\Phi_{2}\right)_{1}}\right) \\
& +8\left(\mu_{12}+\alpha\left(\omega-\omega_{c}\right)\right) \operatorname{Im}\left(F_{\left(\Phi_{1}\right)_{1}}\right)-8 g^{2} i_{2}^{\prime} D_{L, 3} \\
& -2 \mu_{2}^{2} i_{2}^{\prime}-4 \mu_{4}^{2}\left(-i_{2} \cos \beta_{4}+r_{2} \sin \beta_{4}\right)-2 \mu_{5}^{2}\left(-i_{1} \cos \beta_{5}+r_{1} \sin \beta_{5}\right) \text {, }
\end{aligned}
$$




$$
\begin{aligned}
\ddot{d} & =2 a \operatorname{Re}\left(F_{\Omega}\right) \bar{d}+2 F_{\bar{\Delta}}\left(m_{\Delta}+a \omega\right), \\
\ddot{\bar{d}} & =2 a \operatorname{Re}\left(F_{\Omega}\right) d+2 F_{\Delta}\left(m_{\Delta}+a \omega\right), \\
\ddot{d_{c}} & =2 a \operatorname{Re}\left(F_{\Omega_{c}}\right) \bar{d}_{c}+2 F_{\bar{\Delta}_{c}}\left(m_{\Delta}+a \omega_{c}\right), \\
\ddot{\bar{d}}_{c} & =2 a \operatorname{Re}\left(F_{\Omega_{c}}\right) d_{c}+2 F_{\Delta_{c}}\left(m_{\Delta}+a \omega_{c}\right) .
\end{aligned}
$$

\section{B Temperature dependent masses of bidoublet Higgs scalars}

In this section we give the field dependent mass matrix of the bidoublet Higgs fields corresponding to the tree level Higgs potential $V_{\text {tree }}=V_{\text {SUSY }}+V_{\text {soft }}$ of the LRSUSY model at zero temperature. Recall that $V_{\text {SUSY }}$ and $V_{\text {soft }}$ were defined in equations (2.15) and (2.16) respectively.

The field dependent mass matrix of the neutral Higgs bidoublet scalars in the left domain in the $r_{1}, i_{1}, r_{2}, i_{2}, r_{1}^{\prime}, i_{1}^{\prime}, r_{2}^{\prime}, i_{2}^{\prime}$ basis is an $8 \times 8$ real symmetric matrix given by

$$
\begin{aligned}
& M_{r_{1}, r_{1}}=M_{i_{1}, i_{1}} \\
& =8\left(\mu_{11}^{2}+\left(\mu_{12}-\alpha M_{R}\right)^{2}\right)-2 \mu_{1}^{2}+12 g^{2}\left(r_{1}^{2}+i_{1}^{2}-\left(r_{1}^{\prime}\right)^{2}-\left(i_{1}^{\prime}\right)^{2}+r_{2}^{2}+i_{2}^{2}-\left(r_{2}^{\prime}\right)^{2}-\left(i_{2}^{\prime}\right)^{2}\right) \\
& M_{r_{2}, r_{2}}=M_{i_{2}, i_{2}} \\
& =8\left(\left(\mu_{12}+\alpha M_{R}\right)^{2}+\mu_{22}^{2}\right)-2 \mu_{2}^{2}+12 g^{2}\left(r_{1}^{2}+i_{1}^{2}-\left(r_{1}^{\prime}\right)^{2}-\left(i_{1}^{\prime}\right)^{2}+r_{2}^{2}+i_{2}^{2}-\left(r_{2}^{\prime}\right)^{2}-\left(i_{2}^{\prime}\right)^{2}\right) \\
& M_{r_{1}^{\prime}, r_{1}^{\prime}}=M_{i_{1}^{\prime}, i_{1}^{\prime}} \\
& =8\left(\mu_{11}^{2}+\left(\mu_{12}+\alpha M_{R}\right)^{2}\right)-2 \mu_{1}^{2}-12 g^{2}\left(r_{1}^{2}+i_{1}^{2}-\left(r_{1}^{\prime}\right)^{2}-\left(i_{1}^{\prime}\right)^{2}+r_{2}^{2}+i_{2}^{2}-\left(r_{2}^{\prime}\right)^{2}-\left(i_{2}^{\prime}\right)^{2}\right) \\
& M_{r_{2}^{\prime}, r_{2}^{\prime}}=M_{i_{2}^{\prime}, i_{2}^{\prime}} \\
& =8\left(\left(\mu_{12}-\alpha M_{R}\right)^{2}+\mu_{22}^{2}\right)-2 \mu_{2}^{2}-12 g^{2}\left(r_{1}^{2}+i_{1}^{2}-\left(r_{1}^{\prime}\right)^{2}-\left(i_{1}^{\prime}\right)^{2}+r_{2}^{2}+i_{2}^{2}-\left(r_{2}^{\prime}\right)^{2}-\left(i_{2}^{\prime}\right)^{2}\right) \\
& M_{r_{1}, i_{1}}=M_{r_{2}, i_{2}}=M_{r_{1}^{\prime}, i_{1}^{\prime}}=M_{r_{2}^{\prime}, i_{2}^{\prime}}=M_{r_{1}, i_{2}}=M_{i_{1}, r_{2}}=M_{r_{1}^{\prime}, i_{2}^{\prime}}=M_{i_{1}^{\prime}, r_{2}^{\prime}}=0 \\
& M_{r_{1}, r_{2}}=8 \mu_{11}\left(\mu_{12}+\alpha M_{R}\right)+8\left(\mu_{12}-\alpha M_{R}\right) \mu_{22} \\
& M_{i_{1}, i_{2}}=8 \mu_{11}\left(\mu_{12}+\alpha M_{R}\right)+8\left(\mu_{12}-\alpha M_{R}\right) \mu_{22} \\
& M_{r_{1}^{\prime}, r_{2}^{\prime}}=8 \mu_{11}\left(\mu_{12}-\alpha M_{R}\right)+8\left(\mu_{12}+\alpha M_{R}\right) \mu_{22} \\
& M_{i_{1}^{\prime}, i_{2}^{\prime}}=8 \mu_{11}\left(\mu_{12}-\alpha M_{R}\right)+8\left(\mu_{12}+\alpha M_{R}\right) \mu_{22} \\
& M_{r_{1}, r_{1}^{\prime}}=-M_{i_{1}, i_{1}^{\prime}}=-4 \mu_{3}^{2} \cos \beta_{3} \\
& M_{r_{1}, i_{1}^{\prime}}=-M_{i_{1}, r_{1}^{\prime}}=-4 \mu_{3}^{2} \sin \beta_{3} \\
& M_{r_{1}, r_{2}^{\prime}}=-M_{i_{1}, i_{2}^{\prime}}=M_{r_{2}, r_{1}^{\prime}}=-M_{i_{2}, i_{1}^{\prime}}=-2 \mu_{5}^{2} \cos \beta_{5}+48 \alpha^{2}\left(r_{1} r_{2}^{\prime}-i_{1} i_{2}^{\prime}-r_{1}^{\prime} r_{2}+i_{1}^{\prime} i_{2}\right) \\
& M_{r_{1}, i_{2}^{\prime}}=M_{i_{1}, r_{2}^{\prime}}=M_{r_{2}, i_{1}^{\prime}}=M_{i_{2}, r_{1}^{\prime}}=-2 \mu_{5}^{2} \sin \beta_{5}+48 \alpha^{2}\left(r_{1} i_{2}^{\prime}+i_{1} r_{2}^{\prime}-r_{1}^{\prime} i_{2}-i_{1}^{\prime} r_{2}\right) \\
& M_{r_{2}, r_{2}^{\prime}}=-M_{i_{2}, i_{2}^{\prime}}=-4 \mu_{4}^{2} \cos \beta_{4} \\
& M_{r_{2}, i_{2}^{\prime}}=-M_{i_{2}, r_{2}^{\prime}}=-4 \mu_{4}^{2} \sin \beta_{4} .
\end{aligned}
$$

The mass matrix of the charged bidoublet Higgs scalars in the left domain in the basis $\hat{r}_{1}=\operatorname{Re}\left(\left(\Phi_{1}\right)_{12}\right), \hat{i}_{1}=\operatorname{Im}\left(\left(\Phi_{1}\right)_{12}\right), \hat{r}_{1}^{\prime}=\operatorname{Re}\left(\left(\Phi_{1}\right)_{21}\right), \hat{i}_{1}^{\prime}=\operatorname{Im}\left(\left(\Phi_{1}\right)_{21}\right), \hat{r}_{2}=\operatorname{Re}\left(\left(\Phi_{2}\right)_{12}\right)$, 
$\hat{i}_{2}=\operatorname{Im}\left(\left(\Phi_{2}\right)_{12}\right), \hat{r}_{2}^{\prime}=\operatorname{Re}\left(\left(\Phi_{2}\right)_{21}\right), \hat{i}_{2}^{\prime}=\operatorname{Im}\left(\left(\Phi_{2}\right)_{21}\right)$ is the same as above. Due to the fine tuning, half of the neutral and half of the charged scalars have low masses near the EW scale and the other half have masses near $M_{R}$. The field dependent mass matrix of the left handed gauge bosons and the field dependent mass of the top quark is the same as in the Standard Model.

The daisy corrected temperature and field dependent mass matrix is defined by $D_{f_{i}, f_{j}}^{T}=$ $M_{f_{i}, f_{j}}+\frac{T^{2}}{12} \Pi_{f_{i}, f_{j}}$. The matrix $\Pi$ restricted to neutral bidoublet scalars is defined as follows:

$$
\begin{aligned}
& \Pi_{r_{1}, r_{1}}=\Pi_{i_{1}, i_{1}}=9 g^{2}, \\
& \Pi_{r_{1}^{\prime}, r_{1}^{\prime}}=\Pi_{i_{1}^{\prime}, i_{1}^{\prime}}=9 g^{2}-6 y_{t, 1}^{2}, \\
& \Pi_{r_{2}, r_{2}}=\Pi_{i_{2}, i_{2}}=9 g^{2}, \\
& \Pi_{r_{2}^{\prime}, r_{2}^{\prime}}=\Pi_{i_{2}^{\prime}, i_{2}^{\prime}}=9 g^{2}-6 y_{t, 2}^{2} .
\end{aligned}
$$

The daisy corrections modify the mass matrix by $O\left(T^{2}\right)$. However, they do not enter the temperature corrections of the masses of the particles which quadratically depend on the field values in equation (3.1).

Putting all this together, we thus conclude that the leading order temperature corrections in equation (3.1) can now be computed from the Debye mass matrix whose matrix element is defined by [39]

$$
D_{f_{i}, f_{j}}=\frac{T^{2}}{24} \sum_{i} n_{i} \frac{1+(-1)^{2 s_{i}}}{2} \frac{\partial^{2}\left(m_{i}(\Phi)\right)^{2}}{\partial f_{i} \partial f_{j}}
$$

where $m_{i}(\Phi)^{2}$ are the eigenvalues of the field dependent mass matrix defined in equation (B.1) above. We use the Debye mass matrix in obtaining the temperature dependence of the masses of the bidoublet Higgs scalars. The field dependent masses turn out to be proportional to the quartic terms in the Higgs potential which are generically around $O\left(g^{2}\right)$ for a supersymmetric model, where $g$ is the $\mathrm{SU}(2)$ gauge coupling. Thus the temperature corrections to the mass matrix elements are, to a good approximation, around $O\left(g^{2} T^{2}\right)$.

Open Access. This article is distributed under the terms of the Creative Commons Attribution License (CC-BY 4.0), which permits any use, distribution and reproduction in any medium, provided the original author(s) and source are credited.

\section{References}

[1] A.D. Sakharov, Violation of CP Invariance, $C$ asymmetry, and baryon asymmetry of the universe, Sov. Phys. Usp. 34 (1991) 392 [Pisma Zh. Eksp. Teor. Fiz. 5 (1967) 32] [JETP Lett. 5 (1967) 24] [INSPIRE].

[2] V.A. Kuzmin, V.A. Rubakov and M.E. Shaposhnikov, On the Anomalous Electroweak Baryon Number Nonconservation in the Early Universe, Phys. Lett. B 155 (1985) 36 [INSPIRE].

[3] K. Kajantie, M. Laine, K. Rummukainen and M.E. Shaposhnikov, Is there a hot electroweak phase transition at $m_{H} \gtrsim m_{W}$ ?, Phys. Rev. Lett. 77 (1996) 2887 [hep-ph/9605288] [INSPIRE]. 
[4] K. Kajantie, M. Laine, K. Rummukainen and M.E. Shaposhnikov, A Nonperturbative analysis of the finite $T$ phase transition in $\mathrm{SU}(2) \times \mathrm{U}(1)$ electroweak theory, Nucl. Phys. $B$ 493 (1997) 413 [hep-lat/9612006] [INSPIRE].

[5] F. Csikor, Z. Fodor and J. Heitger, Endpoint of the hot electroweak phase transition, Phys. Rev. Lett. 82 (1999) 21 [hep-ph/9809291] [inSPIRE].

[6] ATLAS collaboration, Observation of a new particle in the search for the Standard Model Higgs boson with the ATLAS detector at the LHC, Phys. Lett. B 716 (2012) 1 [arXiv: 1207.7214] [INSPIRE].

[7] CMS collaboration, Observation of a New Boson at a Mass of $125 \mathrm{GeV}$ with the CMS Experiment at the LHC, Phys. Lett. B $\mathbf{7 1 6}$ (2012) 30 [arXiv:1207.7235] [INSPIRE].

[8] M.B. Gavela, P. Hernández, J. Orloff, O. Pene and C. Quimbay, Standard model CP-violation and baryon asymmetry. Part 2: Finite temperature, Nucl. Phys. B 430 (1994) 382 [hep-ph/9406289] [INSPIRE].

[9] R.N. Mohapatra and G. Senjanović, Neutrino Masses and Mixings in Gauge Models with Spontaneous Parity Violation, Phys. Rev. D 23 (1981) 165 [inSPIRE].

[10] R. Kuchimanchi and R.N. Mohapatra, No parity violation without R-parity violation, Phys. Rev. D 48 (1993) 4352 [hep-ph/9306290] [INSPIRE].

[11] C.S. Aulakh, K. Benakli and G. Senjanović, Reconciling supersymmetry and left-right symmetry, Phys. Rev. Lett. 79 (1997) 2188 [hep-ph/9703434] [INSPIRE].

[12] C.S. Aulakh, A. Melfo, A. Rasin and G. Senjanović, Supersymmetry and large scale left-right symmetry, Phys. Rev. D 58 (1998) 115007 [hep-ph/9712551] [INSPIRE].

[13] T.W.B. Kibble, Topology of Cosmic Domains and Strings, J. Phys. A 9 (1976) 1387 [INSPIRE].

[14] T.W.B. Kibble, Some Implications of a Cosmological Phase Transition, Phys. Rept. 67 (1980) 183 [INSPIRE].

[15] J.M. Cline, U.A. Yajnik, S.N. Nayak and M. Rabikumar, Transient domain walls and lepton asymmetry in the left-right symmetric model, Phys. Rev. D 66 (2002) 065001 [hep-ph/0204319] [INSPIRE].

[16] A. Sarkar, Abhishek and U.A. Yajnik, PeV scale left-right symmetry and baryon asymmetry of the Universe, Nucl. Phys. B 800 (2008) 253 [arXiv:0710.5410] [INSPIRE].

[17] L. Canetti, M. Drewes and M. Shaposhnikov, Matter and Antimatter in the Universe, New J. Phys. 14 (2012) 095012 [arXiv: 1204.4186] [InSPIRE].

[18] Y. Yamaguchi and N. Yamanaka, Large long-distance contributions to the electric dipole moments of charged leptons in the standard model, Phys. Rev. Lett. 125 (2020) 241802 [arXiv:2003.08195] [INSPIRE].

[19] ACME collaboration, Improved limit on the electric dipole moment of the electron, Nature 562 (2018) 355 [INSPIRE].

[20] J.F. Nieves, D. Chang and P.B. Pal, Electric Dipole Moment of the Electron in Left-right Symmetric Theories, Phys. Rev. D 33 (1986) 3324 [InSPIRE].

[21] F. Xu, H. An and X. Ji, Neutron Electric Dipole Moment Constraint on Scale of Minimal Left-Right Symmetric Model, JHEP 03 (2010) 088 [arXiv:0910.2265] [InSPIRE]. 
[22] M. Frank, Electric dipole moment of the electron in the left-right supersymmetric model, Phys. Rev. D 59 (1999) 055006 [inSPIRE].

[23] M. Frank, The electric dipole moment of the neutron in the left-right supersymmetric model, J. Phys. G 25 (1999) 1813 [inSPIRE].

[24] S.M. Barr and A. Zee, Electric Dipole Moment of the Electron and of the Neutron, Phys. Rev. Lett. 65 (1990) 21 [Erratum ibid. 65 (1990) 2920] [INSPIRE].

[25] N. Chen, T. Li, Z. Teng and Y. Wu, Collapsing domain walls in the two-Higgs-doublet model and deep insights from the EDM, JHEP 10 (2020) 081 [arXiv:2006.06913] [INSPIRE].

[26] B. Bajc, A. Melfo, G. Senjanović and F. Vissani, The Minimal supersymmetric grand unified theory. 1. Symmetry breaking and the particle spectrum, Phys. Rev. D 70 (2004) 035007 [hep-ph/0402122] [INSPIRE].

[27] D. Chang, R.N. Mohapatra and M.K. Parida, Decoupling Parity and SU $(2)_{R}$-Breaking Scales: A New Approach to Left-Right Symmetric Models, Phys. Rev. Lett. 52 (1984) 1072 [INSPIRE].

[28] J. Chakrabortty, R. Maji and S.F. King, Unification, Proton Decay and Topological Defects in non-SUSY GUTs with Thresholds, Phys. Rev. D 99 (2019) 095008 [arXiv:1901.05867] [INSPIRE].

[29] U. Aydemir, T. Mandal and S. Mitra, Addressing the $R_{D^{(*)}}$ anomalies with an $S_{1}$ leptoquark from $\mathrm{SO}(10)$ grand unification, Phys. Rev. D 101 (2020) 015011 [arXiv:1902.08108] [INSPIRE].

[30] R.N. Mohapatra, G. Yan and Y. Zhang, Ameliorating Higgs induced flavor constraints on TeV scale $W_{R}$, Nucl. Phys. B 948 (2019) 114764 [arXiv: 1902.08601] [INSPIRE].

[31] S. Mishra and U.A. Yajnik, Spontaneously broken parity and consistent cosmology with transitory domain walls, Phys. Rev. D 81 (2010) 045010 [arXiv:0911.1578] [INSPIRE].

[32] M. Hindmarsh, Analytic scaling solutions for cosmic domain walls, Phys. Rev. Lett. 77 (1996) 4495 [hep-ph/9605332] [INSPIRE].

[33] A. Vilenkin and E.P.S. Shellard, Cosmic Strings and Other Topological Defects, Cambridge University Press (2000).

[34] L. Dolan and R. Jackiw, Symmetry Behavior at Finite Temperature, Phys. Rev. D 9 (1974) 3320 [INSPIRE].

[35] D.E. Morrissey and M.J. Ramsey-Musolf, Electroweak baryogenesis, New J. Phys. 14 (2012) 125003 [arXiv: 1206.2942 ] [INSPIRE].

[36] M. Quirós, Finite temperature field theory and phase transitions, in ICTP Summer School in High-Energy Physics and Cosmology, (1999) [hep-ph/9901312] [INSPIRE].

[37] P.B. Arnold and O. Espinosa, The Effective potential and first order phase transitions: Beyond leading-order, Phys. Rev. D 47 (1993) 3546 [Erratum ibid. 50 (1994) 6662] [hep-ph/9212235] [INSPIRE].

[38] P. Basler and M. Mühlleitner, BSMPT (Beyond the Standard Model Phase Transitions): A tool for the electroweak phase transition in extended Higgs sectors, Comput. Phys. Commun. 237 (2019) 62 [arXiv: 1803. 02846] [INSPIRE].

[39] J.M. Cline, K. Kainulainen and M. Trott, Electroweak Baryogenesis in Two Higgs Doublet Models and B meson anomalies, JHEP 11 (2011) 089 [arXiv:1107.3559] [INSPIRE]. 
[40] L. Fromme, S.J. Huber and M. Seniuch, Baryogenesis in the two-Higgs doublet model, JHEP 11 (2006) 038 [hep-ph/0605242] [INSPIRE].

[41] C. Wainwright, Cosmotransitions: computing cosmological phase transition temperatures and bubble profiles with multiple fields, Comput. Phys. Comm. 183 (2012) 2006.

[42] D. Chang, W.-Y. Keung and T.C. Yuan, Two loop bosonic contribution to the electron electric dipole moment, Phys. Rev. D 43 (1991) R14 [InSPIRE].

[43] R.N. Mohapatra and X. Zhang, Electroweak baryogenesis in left-right symmetric models, Phys. Rev. D 46 (1992) 5331 [inSPIRE].

[44] J.M. Frere, L. Houart, J.M. Moreno, J. Orloff and M. Tytgat, Generation of the baryon asymmetry of the universe within the left-right symmetric model, Phys. Lett. B 314 (1993) 289 [hep-ph/9301228] [INSPIRE].

[45] P. Banerjee and U.A. Yajnik, New ultraviolet operators in supersymmetric $\mathrm{SO}(10)$ GUT and consistent cosmology, Phys. Rev. D 101 (2020) 075041 [arXiv:1812.11475] [INSPIRE].

[46] M. Joyce, T. Prokopec and N. Turok, Nonlocal electroweak baryogenesis. Part 2: The Classical regime, Phys. Rev. D 53 (1996) 2958 [hep-ph/9410282] [INSPIRE].

[47] J.M. Cline, M. Joyce and K. Kainulainen, Supersymmetric electroweak baryogenesis, JHEP 07 (2000) 018 [hep-ph/0006119] [INSPIRE].

[48] I. Esteban, M.C. Gonzalez-Garcia, M. Maltoni, T. Schwetz and A. Zhou, The fate of hints: updated global analysis of three-flavor neutrino oscillations, JHEP 09 (2020) 178 [arXiv: 2007.14792] [INSPIRE].

[49] D. Parno, The KATRIN search for neutrino mass, in APS April Meeting, Mini-Symposium: Neutrino Properties: New Developments, Challenges and Impacts, (2021).

[50] C. Grojean and G. Servant, Gravitational Waves from Phase Transitions at the Electroweak Scale and Beyond, Phys. Rev. D 75 (2007) 043507 [hep-ph/0607107] [InSPIRE].

[51] R. Durrer, Gravitational waves from cosmological phase transitions, J. Phys. Conf. Ser. 222 (2010) 012021 [arXiv: 1002.1389] [INSPIRE].

[52] V. Brdar, L. Graf, A.J. Helmboldt and X.-J. Xu, Gravitational Waves as a Probe of Left-Right Symmetry Breaking, JCAP 12 (2019) 027 [arXiv: 1909.02018] [INSPIRE].

[53] M. Li, Q.-S. Yan, Y. Zhang and Z. Zhao, Prospects of gravitational waves in the minimal left-right symmetric model, JHEP 03 (2021) 267 [arXiv: 2012.13686] [INSPIRE].

[54] K. Saikawa, A review of gravitational waves from cosmic domain walls, Universe 3 (2017) 40 [arXiv: 1703.02576] [INSPIRE].

[55] B.P. Abbott et al., Sensitivity of the Advanced LIGO detectors at the beginning of gravitational wave astronomy, Phys. Rev. D 93 (2016) 112004 [Addendum ibid. 97 (2018) 059901] [arXiv: 1604.00439] [INSPIRE].

[56] P. Amaro-Seoane et al., eLISA/NGO: Astrophysics and cosmology in the gravitational-wave millihertz regime, $G W$ Notes 6 (2013) 4 [arXiv:1201.3621] [INSPIRE].

[57] J.D. Wells, PeV-scale supersymmetry, Phys. Rev. D 71 (2005) 015013 [hep-ph/0411041] [INSPIRE].

[58] M. Dhuria and V. Rentala, PeV scale Supersymmetry breaking and the IceCube neutrino flux, JHEP 09 (2018) 004 [arXiv:1712.07138] [INSPIRE]. 